\title{
Understanding High-Gain Twin-Beam Sources Using Cascaded Stimulated Emission
}

\author{
Gil Triginer@, ${ }^{1, \dagger}$ Mihai D. Vidrighin, ${ }^{1,{ }^{*}, \dagger}$ Nicolás Quesada, ${ }^{2, \$}$ Andreas Eckstein, ${ }^{1}$ Merritt Moore, ${ }^{1}$ \\ W. Steven Kolthammer, ${ }^{3}$ J. E. Sipe, ${ }^{4}$ and Ian A. Walmsley ${ }^{1,3}$ \\ ${ }^{1}$ Clarendon Labs, Department of Physics, Oxford University, \\ Parks Road, OX1 3PU Oxford, United Kingdom \\ ${ }^{2}$ Perimeter Institute for Theoretical Physics, Waterloo, Ontario, N2L 2Y5, Canada \\ ${ }^{3}$ Department of Physics, Blackett Laboratory, Imperial College London, \\ London SW7 2AZ, United Kingdom \\ ${ }^{4}$ Department of Physics, University of Toronto, Toronto, Ontario, M5S 1A7, Canada
}

(Received 17 February 2020; revised 11 August 2020; accepted 13 August 2020; published 21 September 2020)

\begin{abstract}
We present a new method for the spectral characterization of pulsed twin-beam sources in the high-gain regime, using cascaded stimulated emission. We show an implementation of this method for a periodically poled potassium titanyl phosphate spontaneous parametric down-conversion source generating up to 60 photon pairs per pulse, and we demonstrate excellent agreement between our experiments and our theory. This work enables the complete and accurate experimental characterization of high-gain effects in parametric down-conversion, including self- and cross-phase modulation. Moreover, our theory allows the exploration of designs with the goal of improving the specifications of twin-beam sources for application in quantum information, computation, sampling, and metrology.
\end{abstract}

DOI: $10.1103 /$ PhysRevX.10.031063

\section{INTRODUCTION}

Many pivotal experiments in quantum optics and technology rely on twin beams generated by sources based on parametric nonlinear optical processes, and in recent years there has been significant progress in the development of such sources. In particular, high-gain two-mode squeezing in modes with well-defined spatial and spectral properties can be produced by parametric down-conversion (PDC) in waveguiding structures, using quasi-phase-matching and group-velocity-matching techniques [1-3]. The generation of twin-beam pulses with up to tens of photon pairs has been demonstrated experimentally [4]. Together with the development of efficient photon-number-resolving detectors [5], this enables a range of new experiments in quantum optics, such as conditional non-Gaussian state preparation [6,7] and boson-sampling experiments [8-11].

In dispersion-engineered pulsed PDC sources, residual spectral correlations between the down-converted beams,

\footnotetext{
* Corresponding author. mihai.vidrighin@gmail.com

${ }^{\dagger}$ G. T. and M. D. V. contributed equally to this work.

Present address: Xanadu, Toronto, Canada.
}

Published by the American Physical Society under the terms of the Creative Commons Attribution 4.0 International license. Further distribution of this work must maintain attribution to the author(s) and the published article's title, journal citation, and DOI.
Subject Areas: Optics, Quantum Physics

due to effects such as phase-matching revivals and nonuniformity in the spectral phase of pump pulses [12-15], result in the generation of a small number of independently squeezed spectral modes with the same spatial profile. In the high-gain regime, self-phase modulation (SPM) of the pump pulses [16,17] and cross-phase modulation (XPM) induced by the pump on the downconverted beams [18] also affect the spectral and spatial structure of PDC emission. In addition, time-ordering corrections in the commonly used Hamiltonian evolution description of PDC must be considered [19,20]. The complex interplay between all of these phenomena makes the study of pulsed PDC sources in the high-gain regime challenging and the development of new techniques for the experimental characterization and theoretical understanding of nonperturbative PDC a priority. This paper focuses on experimental characterization, while in a companion paper [21] we develop theoretical approaches to treat the nonperturbative regime. While the deviations from a simpler framework are particularly well evidenced in the high-gain regime of twin-beam generation, comprehensive modeling and characterization building on our work can be fruitfully applied to many other important applications, such as single-photon sources in integrated photonics for linear optical quantum computing [22] and single-photon frequency conversion [23].

A large body of work has already been devoted to the characterization of PDC sources, of course. Stimulated emission tomography (SET) [24] is a general approach used to infer the quantum properties of a spontaneous 
nonlinear process, such as spontaneous PDC, from an intensity measurement of its corresponding stimulated process, in this example, difference frequency generation (DFG). While it has been the basis of many experiments [25-30] and provides a detailed description of the spectral mode structure of a PDC process, it is generally not used to estimate the degree of squeezing. Although, in principle, it is possible to derive the squeezing strength from the amount of parametric amplification on a seed beam, in practice this method is hindered by uncertainty in the amount of overlap-spatial, spectral, or polarizationbetween the seeding and amplified modes, as well as by optical loss.

An alternative approach consists in using higher-order photon correlation functions of the down-converted field to estimate the number of modes in the source $[19,31,32]$. It has the advantage of being insensitive to loss, but it provides little information about the physical mechanisms that degrade the performance of the source.

Here, we demonstrate a method that provides detailed spectral information about the generated nonclassical light and is applicable in the high-gain regime. It extends SET to the measurement of all emission processes resulting from a second-order optical nonlinearity. Broadband light is generated in the seed mode, effectively seeded through a cascaded process by the stimulated emission signal (see Fig. 1), so the resulting output intensities scale differently with squeezing strength. The ratio between the output intensities can then be used to estimate squeezing parameters, without any knowledge of detection efficiencies or mode overlaps, in a strategy that resembles self-referenced methods for estimating absolute detector efficiency using light generation [33,34]. Additionally, the output of the second process - cascaded stimulated emission—provides phase information due to the coherent addition of light generated by different pump frequencies and can be used to estimate the joint spectral phase of the source.

To understand our cascaded stimulated emission measurements, we use simulations of the PDC process, with a small number of parameters extracted from experiments mainly in the low-gain regime. We rely on Heisenberg-like equations of motion describing the spatial evolution of field amplitudes in the PDC source. Within the undepleted classical pump approximation, we introduce an efficient method for solving these equations with arbitrarily high gain. We find excellent agreement between our experiments and our theoretical predictions. We find that in the largegain regime ( $>2$ photons/pulse spontaneous emission), the performance of the twin-beam generation is significantly affected by nonperturbative evolution, as well as SPM of the pump field and XPM between the pump and the down-converted modes. It has been suggested before that these effects may significantly modify twin-beam source performance $[4,15,20]$, but their impact has not been observed and modeled accurately before. (a)

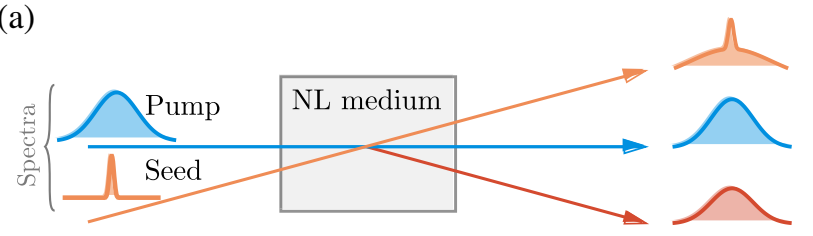

(b.1)

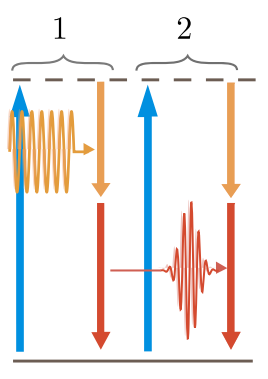

(b.2)
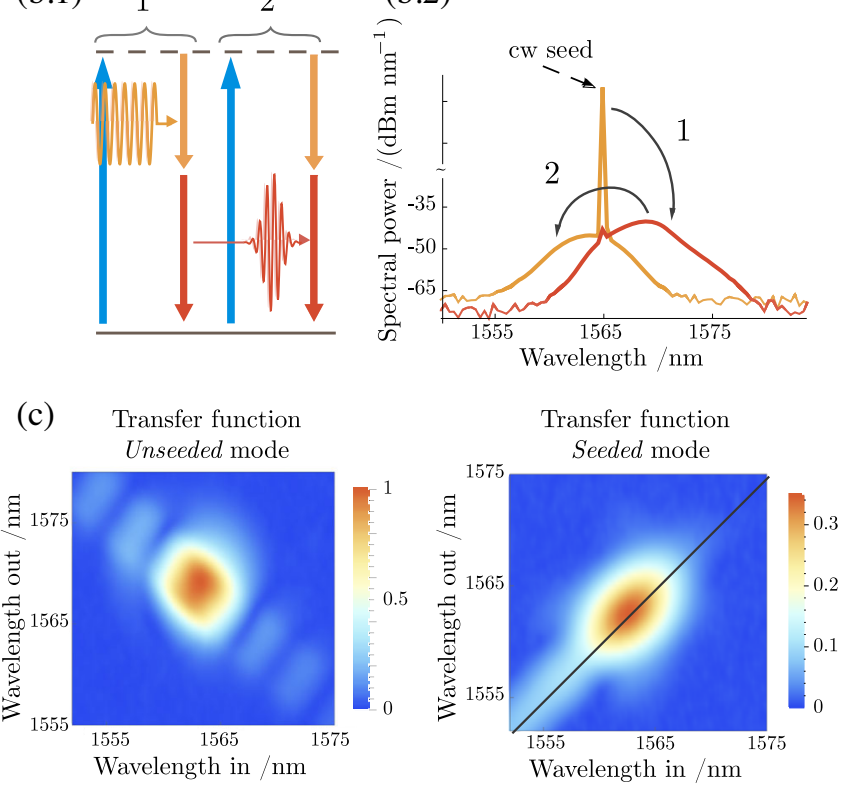

FIG. 1. (a) Schematic of an extended SET experiment, including the broadband light generated in the seeding beam through a cascaded DFG process. (b.1) Cascaded stimulated emission in a PDC crystal. Seeding one of the two down-conversion modes with a continuous-wave (cw) field (represented in orange), bright light is generated through DFG between the pump (represented in blue) and the seed. The DFG signal (represented in red) acts as a seed for a second DFG interaction with the pump field, leading to broadband light generation in the mode that was originally seeded. The process is repeated with decreasing strength. (b.2) Measured spectra of cascaded stimulated emission for a nearly degenerate type-II PDC source pumped with a pulse train centered at $783 \mathrm{~nm}$. Seeding with a $\mathrm{cw}$ laser at $1565 \mathrm{~nm}$, we measured broadband signals in the opposite polarization (red) as well as in the same polarization as the seed (orange). (c) Full spectral response of the PDC process reconstructed by scanning the seed wavelength. The $\mathrm{cw}$ seed contribution was subtracted from the spectra. The spectral responses are normalized to the maximum of the TF corresponding to the unseeded mode.

As our equations describe the PDC source at the quantum level, we are able to accurately quantify the effects of these processes on the generated squeezed vacuum. Our results clarify some previously reported measurements [4] where both nonperturbative effects and third-order processes were present. We also show that our model can be used to optimize the design of sources with the goal of improving figures of merit, such as spectral purity. 
The outline of this paper is as follows: In Sec. I, we introduce the spectral transfer functions that can be used to characterize pulsed squeezing and discuss how they can be extracted from cascaded stimulated emission. Then, we give a theoretical framework for calculating parametric down-conversion, valid both in the limits of small and large gain, and discuss how most parameters for our theoretical description are extracted from experiments in the smallgain regime. In Sec. II, we compare theory and experiment in both the small- and large-gain limits. We discuss our results, and look forward to future experiments, in Sec. III. Further technical details are presented in the Appendixes.

\section{SPECTRAL DESCRIPTION OF PULSED TWIN BEAMS}

\section{A. Input-output relations}

Assuming negligible propagation losses - and within the undepleted, classical pump approximation-broadband two-mode squeezing in a waveguide source supporting a single spatial mode for each field is fully described by frequency-resolved input-output relations [14,15,21]:

$$
\begin{aligned}
a_{\mathrm{s}}^{(\text {out })}(\omega)= & \int d \omega^{\prime} U^{\mathrm{s}, \mathrm{s}}\left(\omega, \omega^{\prime}\right) a_{\mathrm{s}}^{(\mathrm{in})}\left(\omega^{\prime}\right) \\
& +\int d \omega^{\prime} U^{\mathrm{s}, \mathrm{i}}\left(\omega, \omega^{\prime}\right) a_{\mathrm{i}}^{\dagger(\mathrm{in})}\left(\omega^{\prime}\right), \\
a_{\mathrm{i}}^{\text {(out })}(\omega)= & \int d \omega^{\prime} U^{\mathrm{i}, \mathrm{i}}\left(\omega, \omega^{\prime}\right) a_{\mathrm{i}}^{(\mathrm{in})}\left(\omega^{\prime}\right) \\
& +\int d \omega^{\prime} U^{\mathrm{i}, \mathrm{s}}\left(\omega, \omega^{\prime}\right) a_{\mathrm{s}}^{\dagger(\text { in })}\left(\omega^{\prime}\right) .
\end{aligned}
$$

Here, $a_{\mathrm{s}(\mathrm{i})}^{(\mathrm{in})}(\omega)$ denotes the annihilation operator for the input signal (idler) field at frequency $\omega$, and $a_{\mathrm{s}(\mathrm{i})}^{(\mathrm{out})}(\omega)$ denotes the annihilation operator for the respective output mode. The integrals run over the relevant bandwidths. Both input and output operators satisfy the canonical commutation relations $\left[a_{x}(\omega), a_{y}^{\dagger}\left(\omega^{\prime}\right)\right]=\delta_{x, y} \delta\left(\omega-\omega^{\prime}\right)$ and $\left[a_{x}(\omega), a_{y}\left(\omega^{\prime}\right)\right]=0$, with $x, y \in\{\mathrm{s}, \mathrm{i}\}$. The assumptions made in order to derive these relations are detailed in our companion paper [21].

The transfer functions (TFs) $U^{\mathrm{s}, \mathrm{s}}, U^{\mathrm{s}, \mathrm{i}}, U^{\mathrm{i}, \mathrm{s}}$, and $U^{\mathrm{i}, \mathrm{i}}$ completely describe the spectral properties of the state generated in the PDC process, and all observable quantities, such as correlation functions, can be predicted using Eq. (1). The cross-mode TFs $U^{\mathrm{s}, \mathrm{i}(\mathrm{i}, \mathrm{s})}$ are sufficient for describing spontaneous PDC, giving the joint spectral amplitude of a photon pair in the low squeezing regime. However, in this work, we show that measuring the samemode TFs $U^{\mathrm{s},(\mathrm{i}, \mathrm{i})}$, which are only accessible in the stimulated regime, provides a redundancy that is very useful for studying experiments involving high pump powers, where new effects, such as SPM of the pump, come into play. In the application presented here, a full set of measurements involving all the TFs is crucial for providing an explanation of the experimental results at high pump powers.

\section{B. Extracting spectral transfer functions from cascaded stimulated emission}

In SET measurements, a monochromatic seed field is coupled to one of the two polarization-orthogonal downconversion spatial modes. In the presence of the pump, this seed generates light in the other mode by parametric amplification, in a process known as DFG. The emission is mapped over a range of seed frequencies, resulting in a joint spectral distribution [24]. This process has previously been used to measure the joint spectral intensity of photonpair emission in the low squeezing regime [25].

We can now describe SET using the notation introduced above. Equation (1) remains valid when the annihilation and creation operators are replaced by classical field amplitudes $\alpha_{\mathrm{s}(\mathrm{i})}(\omega)$ and their conjugates, as we detail in Appendix A. Therefore, if the idler mode is seeded with the coherent amplitude $\alpha_{i}^{\text {in }}(\omega)$, light will be generated in the signal mode with the amplitude

$$
\alpha_{\mathrm{s}}^{\text {(out) }}(\omega)=\int d \omega^{\prime} U^{\mathrm{s}, \mathrm{i}}\left(\omega, \omega^{\prime}\right) \alpha_{\mathrm{i}}^{*(\mathrm{in})}\left(\omega^{\prime}\right) .
$$

For large $\alpha_{i}^{(\text {in) }}$, the coherent component at the output dominates over the spontaneous emission, and the measured power spectral density (PSD) in the signal mode is $\left|\alpha_{s}^{\text {(out) }}(\omega)\right|^{2}$, with units of photon number per hertz. In Appendix B, we show that, for low PDC gains, the amplitude of $U^{s, i(i, s)}$ scales linearly with a parametric gain proportional to the pump amplitude, as well as to the crystal nonlinearity.

We can retrieve the absolute value of this transfer function using a narrow-band seed at the tunable frequency $\omega_{0}$, such that the PSD at the output is proportional to $\left|U^{\mathrm{s}, \mathrm{i}}\left(\omega, \omega_{0}\right)\right|^{2}$. The proportionality constant is given by the seed intensity, the detection efficiency, and the overlap between the seed mode and the seeded down-conversion mode. Stacking the output spectra measured for a range of seed frequencies, a two-dimensional distribution proportional to $\left|U^{s, i}\left(\omega, \omega^{\prime}\right)\right|^{2}$ can be obtained.

According to the classical equivalent of Eq. (A1), in the seeded measurement, light is also generated in the same mode as the seed, with amplitude

$$
\alpha_{\mathrm{i}}^{(\text {out })}(\omega)=\int d \omega^{\prime} U^{\mathrm{i}, \mathrm{i}}\left(\omega, \omega^{\prime}\right) \alpha_{\mathrm{i}}^{(\mathrm{in})}\left(\omega^{\prime}\right) .
$$

For low gain (see Ref. [13] and Appendix B), $U^{\mathrm{i}, \mathrm{i}}\left(\omega, \omega^{\prime}\right) \approx \delta\left(\omega-\omega^{\prime}\right)$, and Eq. (3) describes linear propagation, without frequency generation in the seed mode. With a more intense pump field, the squeezing strength 
increases, and a broadband "pedestal" is generated at frequencies around the seed frequency. In Appendix B, we show that, in a series expansion of the TFs up to second order in the PDC interaction strength, the broadband contribution to the same-mode TF arises with the term proportional to the square of the parametric gain. A simple physical picture to understand the broadband pedestal is that of cascaded stimulated emission. The DFG field generated in the signal mode leads to the generation of a new DFG field in the idler mode (see Fig. 1).

In a manner analogous to the cross-mode transfer function reconstruction, a two-dimensional distribution proportional to $\left|U^{\mathrm{i}, \mathrm{i}}\right|^{2}$ can be obtained by measuring the spectral intensity in the idler mode for a range of idler seed frequencies. The two remaining transfer functions are obtained by scanning a narrow-band seed in the signal mode and measuring the spectral intensities in the idler $\left(\left|U^{\mathrm{i}, \mathrm{s}}\right|^{2}\right)$ and signal $\left(\left|U^{\mathrm{s}, \mathrm{s}}\right|^{2}\right)$ modes. We denote the broadband part of a same-mode TF as $U_{(\mathrm{b})}^{\mathrm{i}, \mathrm{i}(\mathrm{s}, \mathrm{s})}\left(\omega, \omega^{\prime}\right)=$ $U^{\mathrm{i}, \mathrm{i}(\mathrm{s}, \mathrm{s})}\left(\omega, \omega^{\prime}\right)-\delta\left(\omega-\omega^{\prime}\right)$. To obtain the broadband part of the TF experimentally, we subtract the seed spectrum, which we measure in the absence of the PDC pump, from the output.

We have identified a classical measurement protocol for reconstructing the spectral transfer function corresponding to the $\hat{a}_{s(i)}^{(\text {in) }}$ term in the Bogoliubov transformation for the amplitude operator $\hat{a}_{s(i)}^{\text {(out) }}$ [Eq. (A1)]. Therefore, we have found a useful connection between experimentally accessible information and the comprehensive theoretical description of the twin-beam source. Note that in the common perturbative description of photon-pair generation, this term is ignored altogether.

The seeded measurements are impacted by the (in general, uncalibrated) coupling efficiency of the seed to the downconversion mode, $\eta_{\text {in, s(i) }}$, as well as by the detection efficiency of the generated outputs, $\eta_{\text {out }, \text { (i) }}$. The stimulated emission from $x$ to $y$, where $x, y \in\{\mathrm{s}, \mathrm{i}\}$, is given by

$$
\eta_{\text {in }, x} \eta_{\text {out }, y}\left|U_{(\mathrm{b})}^{y, x}\right|^{2}
$$

where the uncalibrated prefactors hinder the retrieval of the absolute magnitude of the TFs and, therefore, the inference of the parametric gain corresponding to the generated twin beams. We rely on the different scaling of the same and cross-mode TFs with the parametric gain in order to extract it. We define a ratio of TF maxima,

$$
\kappa=\frac{\max \left[\left|U_{(\mathrm{b})}^{\mathrm{s}, \mathrm{s}}\left(\omega, \omega^{\prime}\right)\right|\right]}{\max \left[\left|U^{\mathrm{s}, \mathrm{i}}\left(\omega, \omega^{\prime}\right)\right|\right]} \frac{\max \left[\left|U_{(\mathrm{b})}^{\mathrm{i}, \mathrm{i}}\left(\omega, \omega^{\prime}\right)\right|\right]}{\max \left[\left|U^{\mathrm{i}, \mathrm{s}}\left(\omega, \omega^{\prime}\right)\right|\right]},
$$

which can be obtained by replacing the TFs by the respective measured stimulated PSDs, as the $\eta_{\text {in }}$ and $\eta_{\text {out }}$ coefficients defined in Eq. (4) cancel out. As we have indicated previously, for low PDC gains, the amplitude of the broadband part of the same-mode TFs grows quadratically with the nonlinear gain, while the amplitude of the crossmode TFs grows linearly: Therefore, the ratio $\kappa$ grows linearly with the parametric gain (for low gain). As we detail in subsequent sections, this different scaling allows us to obtain the PDC gain from $\kappa$, independently of the seeding and detection efficiencies, $\eta_{\text {in,s(i) }}$ and $\eta_{\text {out, s(i) }}$.

\section{Theory of parametric down-conversion}

We limit our theoretical treatment to waveguide sources with a single transverse spatial mode for each of the signal, idler, and pump fields. The spectral structure of PDC in such a scenario has been studied before in the perturbative [35] and nonperturbative [13-15,20,36] regimes. We follow an approach close to that of Wasilewski and Lvovsky $[13,14]$, describing the evolution of fields in space rather than in time. At high pump powers, one expects that SPM of the pump and XPM induced by the pump on the signal and idler must be considered, and indeed we find these effects significant in our KTP source. In our companion paper [21], we provide a derivation of the equations of motion (EOMs) of the signal and idler annihilation operators, with these effects included. In a medium where group-velocity dispersion can be ignored within the bandwidth of the signal and idler modes, it is convenient to use a frame of reference that propagates at the group velocity of the pump beam [21,37]. Assuming lossless propagation and an undepleted classical pump, the monochromatic annihilation operators for the slowly varying envelopes of the signal (idler) in this propagating frame of reference, $a_{\mathrm{s}(\mathrm{i})}(z, \omega)$, fulfill the following EOMs [21,37]:

$$
\begin{aligned}
\partial_{z} a_{\mathrm{s}}(z, \omega)= & i \Delta k_{\mathrm{s}}(\omega) a_{\mathrm{s}}(z, \omega) \\
& +i \gamma_{\mathrm{PDC}} g(z)(2 \pi)^{-1 / 2} \int d \omega^{\prime} \beta_{\mathrm{p}}\left(z, \omega+\omega^{\prime}\right) a_{\mathrm{i}}^{\dagger}\left(z, \omega^{\prime}\right) \\
& +i \gamma_{\mathrm{XPM}, \mathrm{s}}(2 \pi)^{-1} \int d \omega^{\prime} \mathcal{E}_{\mathrm{p}}\left(\omega-\omega^{\prime}\right) a_{\mathrm{s}}\left(z, \omega^{\prime}\right),
\end{aligned}
$$

$$
\begin{aligned}
\partial_{z} a_{\mathrm{i}}(z, \omega)= & i \Delta k_{\mathrm{i}}(\omega) a_{\mathrm{i}}(z, \omega) \\
& +i \gamma_{\mathrm{PDC}} g(z)(2 \pi)^{-1 / 2} \int d \omega^{\prime} \beta_{\mathrm{p}}\left(z, \omega+\omega^{\prime}\right) a_{\mathrm{S}}^{\dagger}\left(z, \omega^{\prime}\right) \\
& +i \gamma_{\mathrm{XPM}, \mathrm{i}}(2 \pi)^{-1} \int d \omega^{\prime} \mathcal{E}_{\mathrm{p}}\left(\omega-\omega^{\prime}\right) a_{\mathrm{i}}\left(z, \omega^{\prime}\right) .
\end{aligned}
$$

The first term on the right-hand side of the EOMs contains $\Delta k_{\mathrm{s}(\mathrm{i})}(\omega)=\left(1 / v_{\mathrm{s}(\mathrm{i})}-1 / v_{\mathrm{p}}\right)\left(\omega-\bar{\omega}_{\mathrm{s}(\mathrm{i})}\right)$. Here, $v_{\mathrm{s}(\mathrm{i})}$ and $v_{\mathrm{p}}$ are the group velocities of the signal (idler) and pump pulses, respectively, and $\bar{\omega}_{\mathrm{s}(\mathrm{i})}$ is the central frequency of the signal (idler) mode. This term in the EOMs effectively describes linear propagation through the waveguide. 
The complex function $\beta_{\mathrm{p}}(z, \omega)$ is the pump spectral amplitude in the chosen reference frame, which evolves slowly along $z$ due to SPM inside the nonlinear region [21], according to

$$
\partial_{z} \beta_{\mathrm{p}}(z, \omega)=i \gamma_{\mathrm{SPM}} \int d \omega^{\prime} \mathcal{E}_{\mathrm{p}}\left(\omega-\omega^{\prime}\right) \beta_{\mathrm{p}}\left(z, \omega^{\prime}\right) .
$$

The pump spectral amplitude satisfies the normalization

$$
\int d \omega\left|\beta_{\mathrm{p}}(z, \omega)\right|^{2}=\mathcal{E}_{\mathrm{p}}(0)=E_{\mathrm{p}},
$$

where $E_{\mathrm{p}}$ is the energy in the pump pulse. Note the difference in units between $\beta_{p}(z, \omega)$ and the $\alpha_{s(i)}(\omega)$ introduced earlier, near Eqs. (2) and (3).

The constant $\gamma_{\text {PDC }}$ in Eqs. (6a) and (6b) is the secondorder nonlinear coupling strength, and the function $g(z)$ accounts for the possibility of a sign reversal of this coefficient as is the case in periodically poled crystals. It takes the values 1 or -1 over the length of a periodically poled region and zero outside the crystal.

The third term describes XPM between the pump and the signal (idler), with $\gamma_{\mathrm{XPM}, \mathrm{s}(\mathrm{i})}$ a coupling strength that can be different for the signal and idler. This term also contains the frequency autocorrelation function of the pump spectral amplitude, which, in this frame of reference, is spatially invariant [21],

$$
\mathcal{E}_{\mathrm{p}}(\Delta \omega)=\int d \omega^{\prime \prime} \beta_{\mathrm{p}}\left(z_{0}, \omega^{\prime \prime}-\Delta \omega\right)^{*} \beta_{\mathrm{p}}\left(z_{0}, \omega^{\prime \prime}\right),
$$

where $z_{0}$ is the input of the waveguide.

The solutions of the EOMs (6a) and (6b) have a special form, which is related to the fact that the commutation relations are preserved [21]. The TFs can always be written as

$$
\begin{aligned}
U^{\mathrm{s}, \mathrm{s}}\left(\omega, \omega^{\prime}\right) & =\sum_{l} \cosh \left(r_{l}\right) \rho_{\mathrm{s}}^{(l)}(\omega) \tau_{\mathrm{s}}^{(l)}\left(\omega^{\prime}\right), \\
U^{\mathrm{s}, \mathrm{i}}\left(\omega, \omega^{\prime}\right) & =\sum_{l} \sinh \left(r_{l}\right) \rho_{\mathrm{s}}^{(l)}(\omega)\left[\tau_{i}^{(l)}\left(\omega^{\prime}\right)\right]^{*}, \\
U^{\mathrm{i}, \mathrm{i}}\left(\omega, \omega^{\prime}\right) & =\sum_{l} \cosh \left(r_{l}\right) \rho_{\mathrm{i}}^{(l)}(\omega) \tau_{\mathrm{i}}^{(l)}\left(\omega^{\prime}\right), \\
U^{\mathrm{i}, \mathrm{s}}\left(\omega, \omega^{\prime}\right) & =\sum_{l} \sinh \left(r_{l}\right) \rho_{\mathrm{i}}^{(l)}(\omega)\left[\tau_{\mathrm{s}}^{(l)}\left(\omega^{\prime}\right)\right]^{*} .
\end{aligned}
$$

Here, $\rho_{s(i)}^{(l)}$ are normalized signal (idler) output spectral modes, $\tau_{\mathrm{s}(\mathrm{i})}^{(l)}$ are the corresponding input modes, and $r_{l}$ are the corresponding squeezing parameters.

Remarkably, if SPM can be neglected and the PDC nonlinearity is uniform or has a homogeneous periodic poling, the EOMs (6a) and (6b) can be efficiently solved by exponentiating the discretized differential operator [21]. Scenarios involving slow spatial variations of either the pump amplitude (through SPM) or the effective nonlinearity in periodically poled crystals require an additional step. In those situations, we split the crystal into a number of sections along the propagation direction, within each of which the pump amplitude can be assumed uniform. The input-output relations are then found by sequentially applying the transformations for all sections. This procedure remains very efficient-for the discretized transformations, it amounts to matrix multiplication-allowing us to perform parameter sweeps effectively even in the high-gain regime.

In treatments of squeezing, it is sometimes assumed that one can neglect both the cross-phase modulation of the signal and idler by the pump, and the SPM of the pump itself. We refer to treatments that make this assumption as " $\chi^{(2)}$ models." In addition, when a weak quadratic nonlinearity is assumed, an analytical perturbative solution can be derived for the joint spectral amplitude. In Ref. [15], a high-gain extrapolation of such a perturbative solution was used as an approximative model, in order to highlight nonperturbative effects in the numerical results. We refer to this as a "simplified $\chi^{(2)}$ model."

The more general description that our EOMs (6a) and (6b) provide goes beyond these models. The solutions of the EOMs recover features of PDC that have been introduced before as the results of time-ordering corrections [20], including an enhanced squeezing rate as a function of the pump power, and the distortion (frequency broadening) of the Schmidt modes [15,20,21]. Moreover, they account for the spectral transformation of the twin beams caused by SPM and XPM. In consequence, we refer to our description as a " $\chi^{(2)} / \chi^{(3)}$ model."

In subsequent sections, we address how all the parameters appearing in the EOMs (6a), (6b), and (7) can be accurately determined, and we experimentally confirm the predictions of the equations.

\section{Extracting physical parameters from experiments}

Our experimental demonstration employs a waveguided periodically poled potassium titanyl phosphate (PPKTP) type-II PDC source. The pump pulses with a central wavelength of $783 \mathrm{~nm}$ and a pulse duration of around 1 ps propagate along the ordinary axis of the crystal $(\mathrm{H}$ polarization). They generate a signal field with the central wavelength at $1563 \mathrm{~nm}$ in the $\mathrm{H}$ polarization and an idler field at $1569 \mathrm{~nm}$ in the $\mathrm{V}$ polarization. More details are provided in Appendix C.

The design, reproduced from earlier work [2], achieves a nearly separable joint spectrum. Therefore, the source generates almost single-mode broadband, orthogonally polarized, twin beams. The waveguide confinement makes the source very bright. Previous to this work, up to 20 


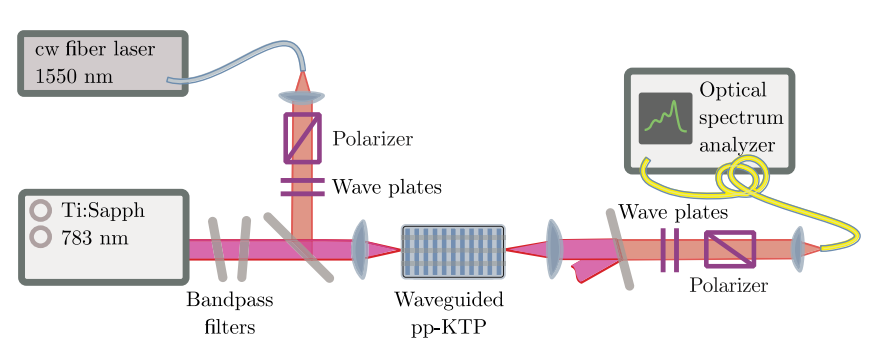

FIG. 2. Experimental setup.

photons per pulse (on average) generated by spontaneous PDC have been reported [4]. However, the spectral structure of the twin beams in the high-gain regime has not been systematically analyzed. Here, we show that we can correctly predict the absolute values of the measured TFs for this source up to an inferred mean photon number of 60 per pulse.

The cascaded stimulated emission setup is depicted in Fig. 2. We scan the frequency of a cw seed laser across the bandwidth of the signal or idler mode. After selecting a down-converted field by polarization filtering, we record the spectrum of the generated light using an optical spectrum analyzer (OSA). We perform measurements for pump pulse energies ranging from $125 \mathrm{pJ}$ to $600 \mathrm{pJ}$. The pulse energy of the generated stimulated emission is measured to be below $0.1 \mathrm{pJ}, 3$ orders of magnitude smaller than that of the pump. This result justifies the nondepleted pump approximation in our models. For each pump power, we obtain all four TFs (absolute value) as explained in Sec. II B.

Most parameters in Eq. (6) can be measured for this source in the low-gain regime, either from stimulated emission data or from simple complementary experiments, as we summarize in this section. The values of the parameters, as well as the methods we used to obtain them, are listed in Table I. The reader interested in replicating our method can find the necessary details in Appendix D.

We measure the pump power spectral amplitude, $\mid \beta_{\mathrm{p}}(z=$ $0, \omega)\left.\right|^{2}$ (Appendix D 1), with an OSA. We extract the spectral phase of the pump, $\arg \left[\beta_{\mathrm{p}}(z=0, \omega)\right]$, using a standard ultrafast spectral phase measurement, SPIDER, for the laser pulse and a spectral self-interference measurement (Appendix D2) for the phase added by the bandpass filters used to shape the pump pulse, as shown in Fig. 2.

We obtain the group-velocity mismatch between pump and signal (idler) pulses, $\left(1 / v_{\mathrm{s}(\mathrm{i})}-1 / v_{\mathrm{p}}\right)$, from the angle and bandwidth of the phase-matching function in a lowgain JSI (Appendix D 3). This result is confirmed by a direct interferometric measurement of the differential delay between the two polarizations (signal or idler) using a broadband field that propagates through the birefringent PPKTP crystal (Appendix D 3).

We extract the PDC interaction strength $\gamma_{\mathrm{PDC}}$ from the ratio between the maxima of the same-mode and crossmode TFs in the low-gain regime, where these amplitudes are not influenced by other nonlinear processes (Appendix D 4). The XPM interaction strength is obtained from the shape of the same-mode $\mathrm{TF}$ in the low-gain regime alone, as we show in the next section, as well as in Appendix D 5. The SPM interaction strength $\gamma_{\text {SPM }}$ is the only parameter that we extract from a fit in the high-gain regime: Once all other parameters are fixed, $\gamma_{\mathrm{SPM}}$ is chosen to optimize the fitting of the high-gain TFs (Appendix D 6).

\section{EXPERIMENTAL DEMONSTRATION}

Here, we report our cascaded stimulated emission measurements for an ample range of gains and demonstrate the excellent agreement with our simulated TFs. Figure 3 summarizes the results for the lowest and highest pump pulse energies. Each measured TF amplitude is compared to the prediction of our EOMs (6a) and (6b), with parameters fitted as explained in detail in Appendix D. Remarkably, a single model is able to accurately account for all the experimental data. In what follows, we report on the most important features of the measured TFs and provide their physical interpretation.

\section{A. Low-gain spectra}

We first measured the cross-mode stimulated emission using a relatively low pump power (125 pJ), reproducing a standard SET experiment. The square roots of the resulting

TABLE I. Model parameters.

\begin{tabular}{lccl}
\hline \hline Parameter & Symbol & Value & \multicolumn{1}{c}{ Method } \\
\hline Pump spectral amplitude & $\left|\beta_{\mathrm{p}}\left(z_{0}, \omega_{\mathrm{p}}\right)\right|$ & $\ldots$ & Optical spectrum analyzer \\
Pump spectral phase & $\arg \left[\beta_{\mathrm{p}}\left(z_{0}, \omega_{\mathrm{p}}\right)\right]$ & $\ldots$ & SPIDER and spectral interferometry \\
Group-velocity mismatch & $1 / v_{\mathrm{s}}-1 / v_{\mathrm{p}}$ & $1.70 \mathrm{ps} / \mathrm{cm}$ & Angle and bandwidth of phase-matching profile \\
& $1 / v_{\mathrm{i}}-1 / v_{\mathrm{p}}$ & $-1.02 \mathrm{ps} / \mathrm{cm}$ & + direct interferometric measurement of group delay \\
PDC coupling & $\gamma_{\mathrm{PDC}}$ & $28 \mathrm{~W}^{-1 / 2} \mathrm{~m}^{-1}$ & Ratio of the cross-mode and same-mode TF amplitudes \\
XPM coupling & $\gamma_{\mathrm{XPM}, \mathrm{s}}$ & $0.16 \mathrm{~W}^{-1} \mathrm{~m}^{-1}$ & Asymmetry of the same-mode TFs \\
& $\gamma_{\mathrm{XPM}, \mathrm{i}}$ & $0.059 \mathrm{~W}^{-1} \mathrm{~m}^{-1}$ & \\
SPM coupling & $\gamma_{\mathrm{SPM}}$ & $0.56 \mathrm{~W}^{-1} \mathrm{~m}^{-1}$ & Fitting of TFs in the high-gain regime \\
\hline \hline
\end{tabular}


(a)
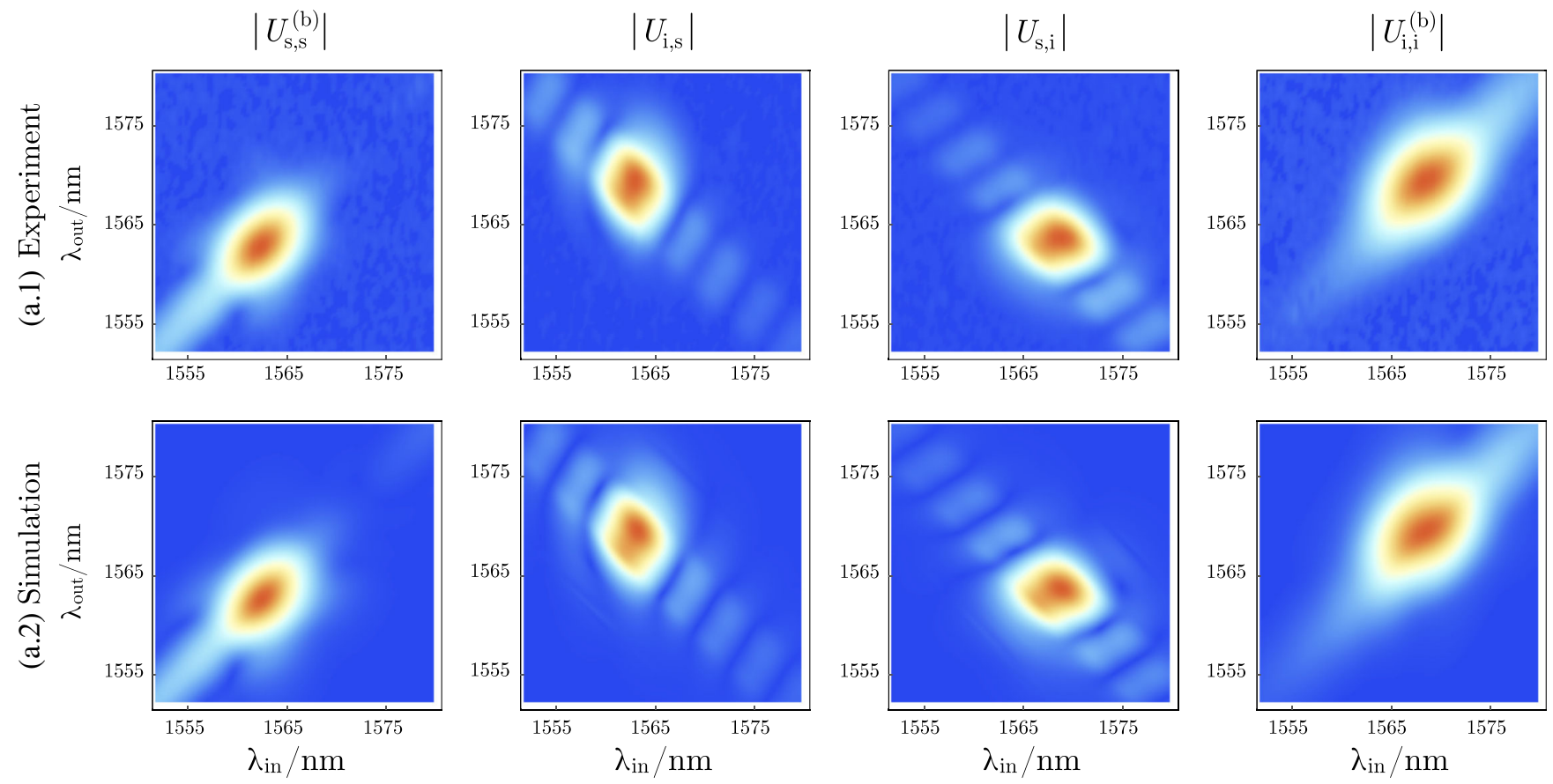

(b)
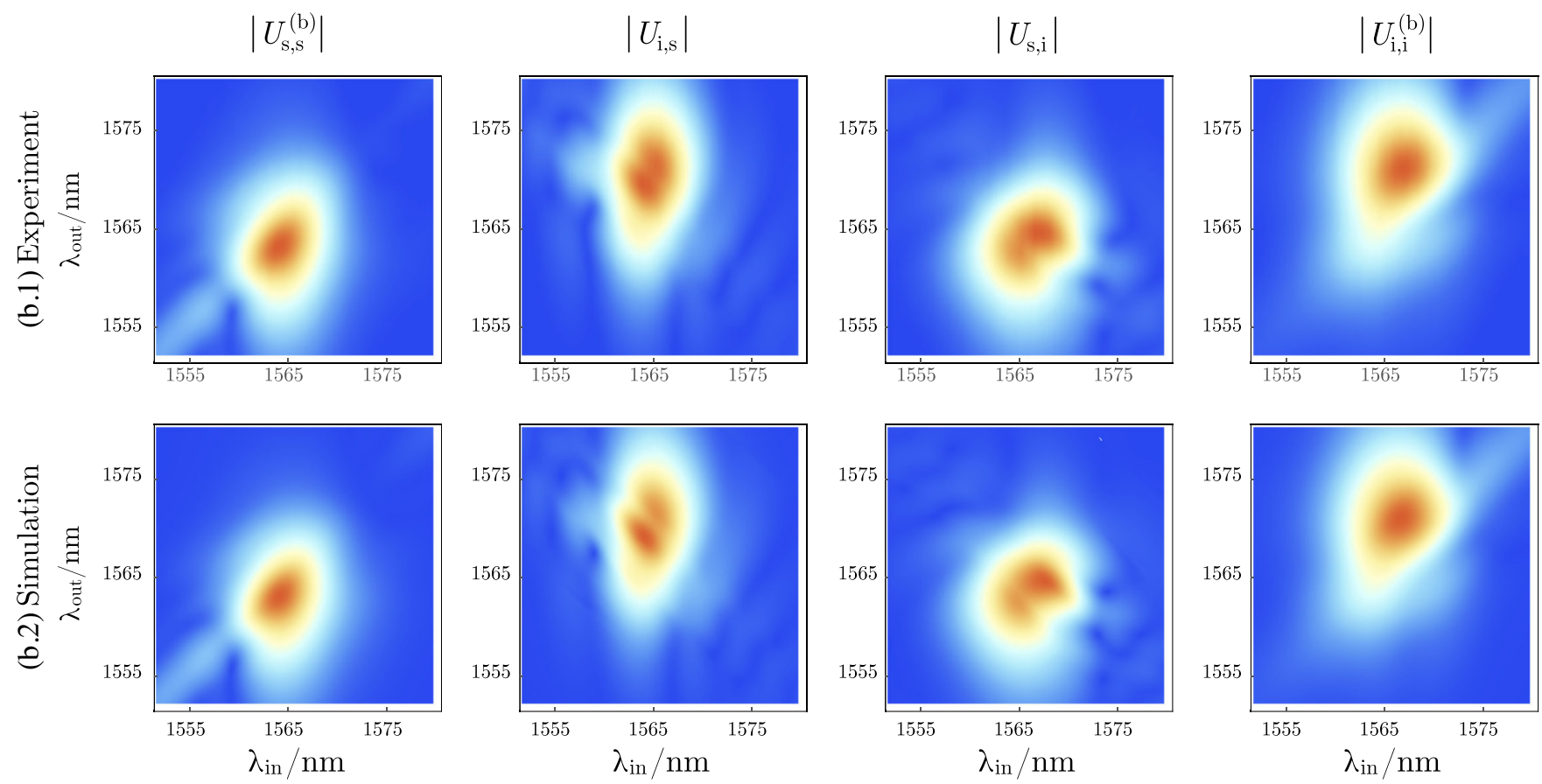

Color scale

\begin{tabular}{|ll}
\hline & $\max / 2$ \\
\hline 0 & $\max$ \\
\hline
\end{tabular}

FIG. 3. All measured and modeled TF amplitudes, in the low- and high-gain regimes, corresponding to strong and weak squeezing. The color scale is normalized separately for each density plot. The TF amplitudes are plotted as a function of wavelength instead of angular frequency, where they are connected by $\lambda=2 \pi c / \omega$. (a) Low squeezing (approx. 2 photons/pulse spontaneous emission) (b) High squeezing (approx. 60 photons/pulse spontaneous emission). 
two-dimensional PSDs, corresponding to the absolute value of $U^{\mathrm{s}, \mathrm{i}}$ and $U^{\mathrm{i}, \mathrm{s}}$, are depicted in the low-gain section of Fig. 3. We inferred a spontaneous emission rate of approximately 2 photons/pulse. In this regime, the crossmode $\mathrm{TF}$ is equal to the joint spectral amplitude of the generated photon pairs.

In the same setup, we were able to resolve broadband stimulated emission in the same polarization as the cw seed, centered around the seed wavelength. By scanning the seed wavelength, we reconstructed the two-dimensional spectral distributions, which are shown in the low-gain section in Fig. 3. As discussed in Sec. II B, these are proportional to the absolute value of the same-mode TFs $U_{(\mathrm{b})}^{\mathrm{s}, \mathrm{s}}$ and $U_{(\mathrm{b})}^{\mathrm{i}, \mathrm{i}}$.

In Fig. 4, we compare experiment and simulation along the $\omega^{\prime} \equiv \omega_{\text {in }}=\omega_{\text {out }} \equiv \omega$ cut of the same-mode TFs. For comparison, we added the prediction of a $\chi^{(2)}$ model. The $\chi^{(2)}$ model is significantly less accurate, failing to predict the asymmetry in the tails of the distribution observed experimentally. We find that this asymmetry is explained by the presence of cross-phase modulation induced by the pump pulse on the down-converted modes. For frequencies far from the PDC phase matching, the effect of XPM appears as a pedestal around the cw seed. Along the $\omega_{\text {in }}=$ $\omega_{\text {out }}$ contour, XPM results in a constant background. The broadband component of the same-mode $\chi^{(2)} \mathrm{TF}$, obtained from our simulations, shows a $\pi$ phase step around the central wavelength. In Appendix B, Eq. (B21), we show, using an analytic approximation, that this $\pi$ phase shift is a general feature. The total TF is a coherent addition of the XPM background and the narrow-band PDC signal, resulting in a Fano resonance [38]. This coherent addition explains the asymmetric shape illustrated in Fig. 4. As indicated above and detailed in Appendix D 5, we extract the XPM interaction strength by optimizing the experiment-theory fit for the profiles in Fig. 4. We find that the XPM interaction strength is not equal for the signal and idler modes, and we analyze this observation in detail in Appendix E. The signal (with the same polarization as the pump) has an XPM interaction strength roughly 3 times greater than the idler (with polarization orthogonal to that of the pump). The asymmetry of the same-mode TFs is observed to be independent of the pump power, as both cascaded PDC and XPM scale quadratically with the pump amplitude in the low-gain regime. The independence of pump power makes the TF asymmetry a robust feature and a good way to estimate the ratio between $\chi^{(2)}$ and $\chi^{(3)}$ interaction strengths.

\section{B. High-gain spectra}

Increasing the pump power, we observe additional deviations from the $\chi^{(2)}$ model discussed above. The high-gain section in Fig. 3 shows measurements with a pump pulse energy of $600 \mathrm{pJ}$; we infer a spontaneous generation rate of about 60 pairs of photons per pump
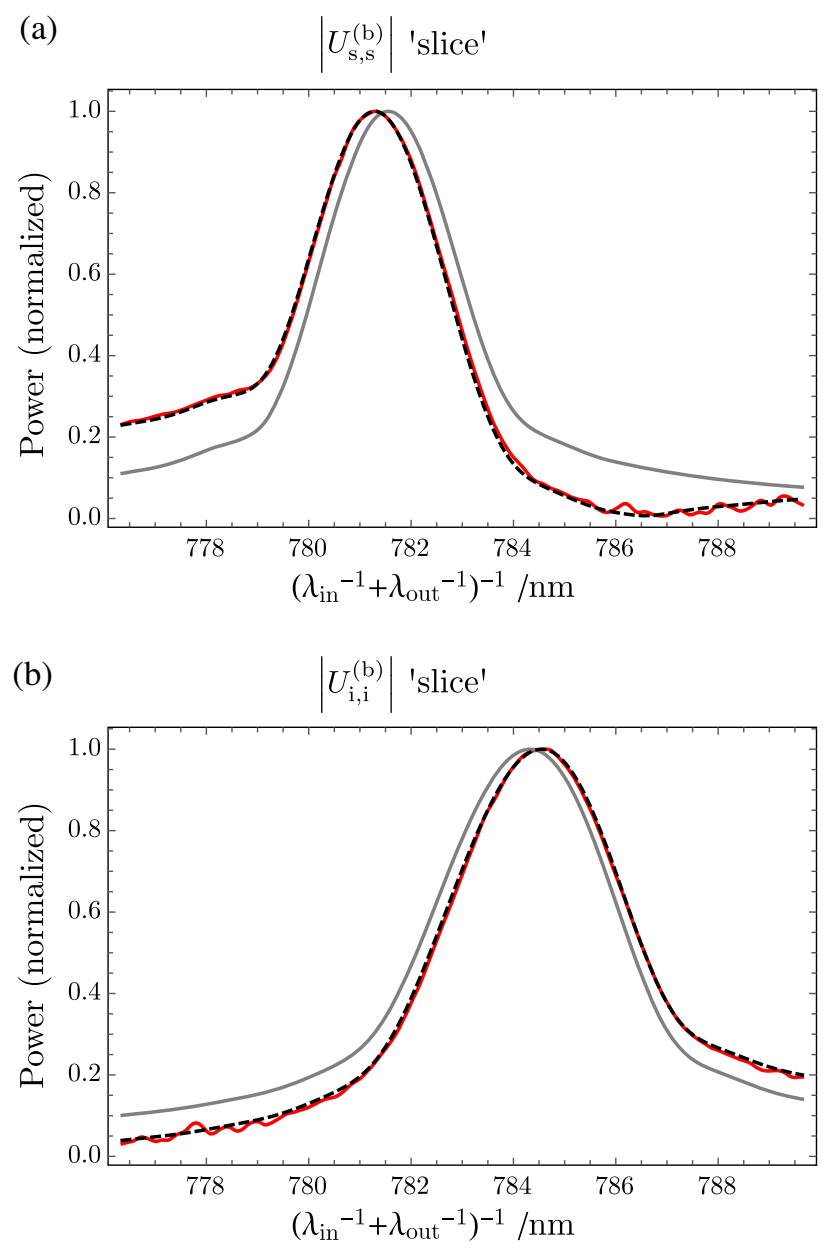

FIG. 4. Same-mode TF slices showing the effect of XPM. The plots show $\left|U_{(\mathrm{b})}^{\mathrm{s}, \mathrm{s}(\mathrm{i}, \mathrm{i})}\left(\lambda_{\text {in }}, \lambda_{\text {out }}\right)\right|$ for $\lambda_{\text {in }}=\lambda_{\text {out }}$. Red lines: Measurement (background subtracted). Black dashed lines: Model prediction. Gray lines: Prediction of a model that does not include XPM. The asymmetry arises from superposing the signals generated by cascaded stimulated PDC and XPM.

pulse. As expected in the high-gain regime, phase matching lobes are suppressed, and the spectra are broadened [19]. In addition to these effects, accounted for by the $\chi^{(2)}$ model, the cross-mode TFs are highly distorted and the same-mode TFs show a significant asymmetry with respect to the $\omega_{\text {in }}=$ $\omega_{\text {out }}$ line.

We can account for these features only by incorporating the effect of self-phase modulation of the pump into Eqs. (6a) and (6b). The excellent agreement between the $\chi^{(2)} / \chi^{(3)}$ model and the high-gain data is clear evidence that SPM explains spectral features that appear with high pump pulse energy. The distortion of the cross-mode TFs is related to the broadening and splitting of the pump spectrum through SPM. The shift of the same-mode TFs can be explained by the chirp accumulated by the pump pulse due to SPM. As illustrated in Fig. 5, the spectral phase of the pump is mapped onto the absolute value of the same-mode TFs, as signals generated by different pump 

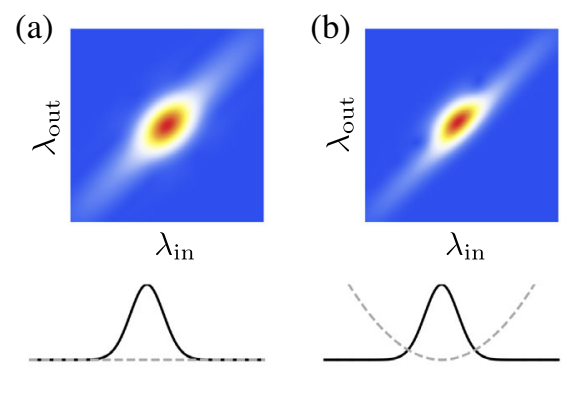

- - - Pump phase

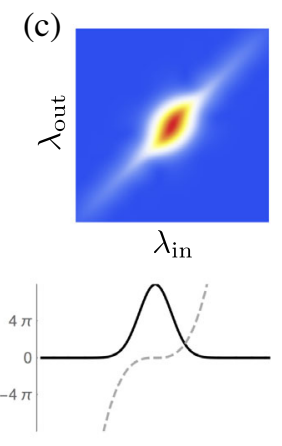

FIG. 5. Amplitudes of the same-mode TFs, $U_{(\mathrm{b}}^{\mathrm{s}, \mathrm{i}(\mathrm{i}, \mathrm{i})}\left(\omega, \omega^{\prime}\right)$. Simulated same-mode TF for (a) Fourier-limited, (b) chirped, and (c) higher-order chirped Gaussian pump pulses (intensity and bandwidth in arbitrary units). A chirped pump causes a displacement of the same-mode TF from the diagonal (b), while a higherorder chirp leads to a more complicated deformation of the same-mode TF.

frequencies add up coherently in the cascaded frequency generation process. In particular, a quadratic phase in the pump field causes a shift of the same-mode TFs away from the $\omega_{\text {in }}=\omega_{\text {out }}$ line.

The fact that changes in the spectral phase of the crossmode transfer function (JSA in the low-gain regime) are mapped onto significant changes of the absolute value of the same-mode transfer functions gives us access to spectral phase information using only intensity measurements. While we do not develop this here, in principle, a phase-retrieval algorithm can be constructed based on this observation.

\section{Scaling and absolute, loss-independent validation}

The different scaling of same- and cross-mode TFs gives us access to the twin-beam gain using cascaded stimulated emission measurements outside of the high-gain regime. As we show in Sec. II B and Appendix D 4, we can use ratios of the measured PSDs to construct the coefficient $\kappa$, defined in Eq. (5), which grows monotonically with the PDC interaction strength and which is independent of detection efficiency and mode overlap. Note that this coefficient captures features related to the gain of the process in both the low- and high gain regimes, and that in the low-gain regime, it gives information pertaining to the squeezing parameters characterizing the transformation of operators in Eq. (10d). Figure 6 shows that our theory correctly predicts the scaling of $\kappa$ observed in the experiments.

\section{Difference between measurement and simulation}

Our theoretical model is consistent with experimental data for a broad range of spontaneous photon-pair generation rates, from 1 photon pair/pulse on average to 60 photon pairs/pulse. To quantify the agreement between experiment and theory, we introduce the integrated squared error between the measured and simulated absolute values of the TFs, normalized to unit area:

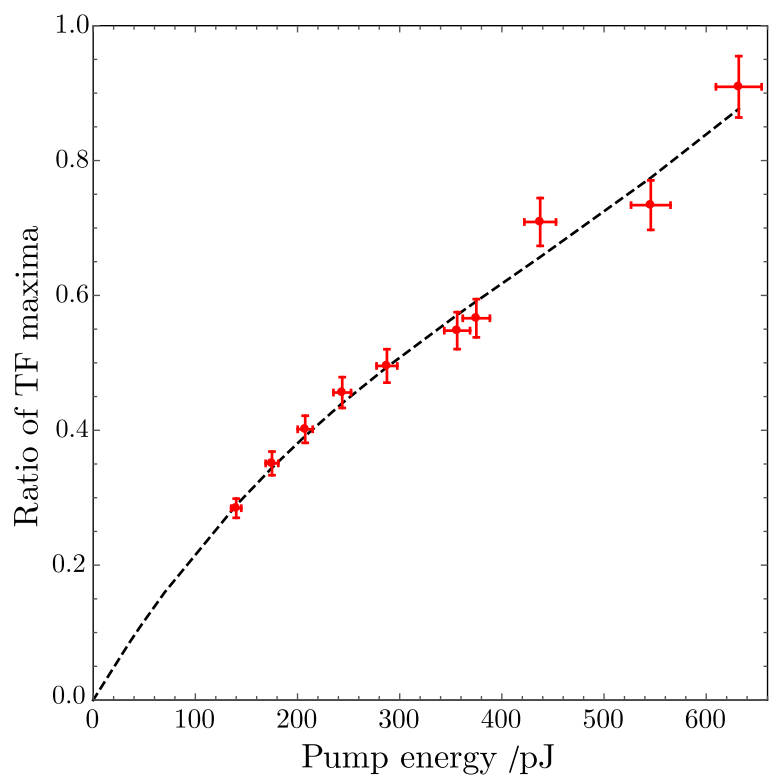

FIG. 6. Ratio between the self- and cross-mode transfer functions $(\kappa)$ for different pump pulse energies.

$$
\begin{aligned}
\varepsilon^{x, y} & =\int d \omega d \omega^{\prime}\left|\frac{\left|U_{\text {meas }(\mathrm{b})}^{x, y}\left(\omega, \omega^{\prime}\right)\right|}{\mathcal{N}_{\text {meas }}^{x, y}}-\frac{\left|U_{\text {sim }(\mathrm{b})}^{x, y}\left(\omega, \omega^{\prime}\right)\right|}{\mathcal{N}_{\text {sim }}^{x, y}}\right|^{2}, \\
\mathcal{N}_{\text {sim }}^{x, y} & =\left[\int d \omega d \omega^{\prime}\left|U_{\text {meas }(\mathrm{b})}^{x, y}\left(\omega, \omega^{\prime}\right)\right|^{2}\right]^{1 / 2}, \\
\mathcal{N}_{\text {sim }}^{x, y} & =\left[\int d \omega d \omega^{\prime}\left|U_{\text {sim }(\mathrm{b})}^{x, y}\left(\omega, \omega^{\prime}\right)\right|^{2}\right]^{1 / 2}, \\
x, y & \in\{\mathrm{s}, \mathrm{i}\} .
\end{aligned}
$$

Here, $U_{\text {meas (b) }}^{x, y}$ is the broadband part of the power spectral density measured in mode $y$ when seeding mode $x$, and $U_{\operatorname{sim}(\mathrm{b})}^{x, y}$ is the corresponding simulated TF. In Fig. 7, we show that, using the calibrated $\chi^{(2)} / \chi^{(3)}$ model (solid lines), $\varepsilon^{x, y}<2 \%$ for all pump powers. On the other hand,

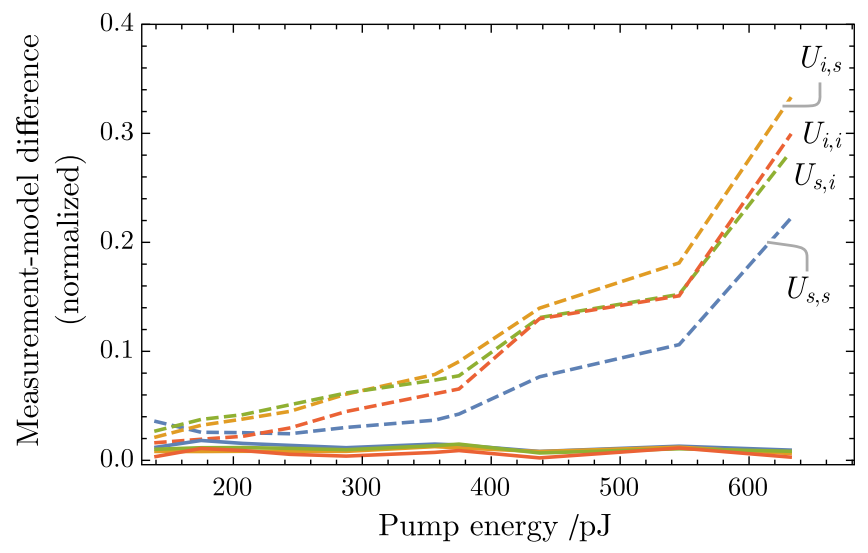

FIG. 7. Mean-squared error between measured and modeled TFs (details in the Sec. III.D). Solid lines are for the $\chi^{(2)} / \chi^{(3)}$ model; dashed lines are for the $\chi^{(2)}$ model. 
neglecting the effect of XPM and SPM (dashed lines), we cannot produce a model that fits the experimental data for all pump powers.

\section{PERFORMANCE OF THE SOURCE}

We have characterized a source designed to provide a high degree of squeezing in a small number of spectral modes. We have developed a theoretical description that is validated by our experimental results. We now use this description to analyze the performance of the source. In particular, we discuss high-gain effects on brightness and spectral purity.

\section{A. Photon number}

We can calculate the spontaneously generated mean photon number by adding the squares of the Schmidt coefficients of the cross-mode TF [15], $\langle n\rangle=\sum_{l} \sinh ^{2}\left(r_{l}\right)$. In the perturbative cross-mode TFs (obtained in Appendix B), the squeezing parameters are linearly proportional to the pump amplitude. In the "simplified $\chi^{(2)}$ model" [15], the spontaneously generated mean photon number is $\langle n\rangle=\sum_{l} \sinh ^{2}\left(c_{l} \sqrt{E_{p}}\right)$, where $E_{\mathrm{p}}$ is the pump pulse energy and $c_{l}$ are constants set by the perturbative solution. Our theoretical approach based on integrating EOMs offers revised predictions, previously treated as time-ordering corrections $[15,20]$ (the connection between our treatment and time domain evolution is clarified in Ref. [21]).

As illustrated in Fig. 8(a), the mean photon number predicted by the $\chi^{(2)}$ model grows significantly faster with pump pulse energy than predicted by the simplified $\chi^{(2)}$ model. The $\chi^{(2)}$ photon number scaling is not simply a $\sinh ^{2}$ function with a different coefficient but rather a different function. The predictions of the different models converge in the low-gain regime but scale differently for higher gain. The accurate mean photon number per pulse is given by the " $\chi^{(2)} / \chi^{(3)}$ model," which combines the effect of nonperturbative SPDC with that of SPM. The opposing directions of these effects result in the nonperturbative corrections being effectively hidden by SPM, such that the brightness of the spontaneous emission can approach that expected from a simplified $\chi^{(2)}$ computation [4]. Our simulations show that the effect of XPM on the mean photon number is negligible.

\section{B. Spectral purity}

We now consider the spectral multimodeness of the source, quantified by the Schmidt number, defined as $K=$ $\left(\sum_{l} \sinh \left(r_{l}\right)^{2}\right)^{2} /\left(\sum_{l} \sinh \left(r_{l}\right)^{4}\right)$ [19,39]. A low Schmidt number implies that there is little correlation between the frequency spread of the signal and idler modes and thus, ultimately, very low spectral entanglement. This situation therefore allows high-purity single photons to be heralded from pairs generated in the low-gain regime.
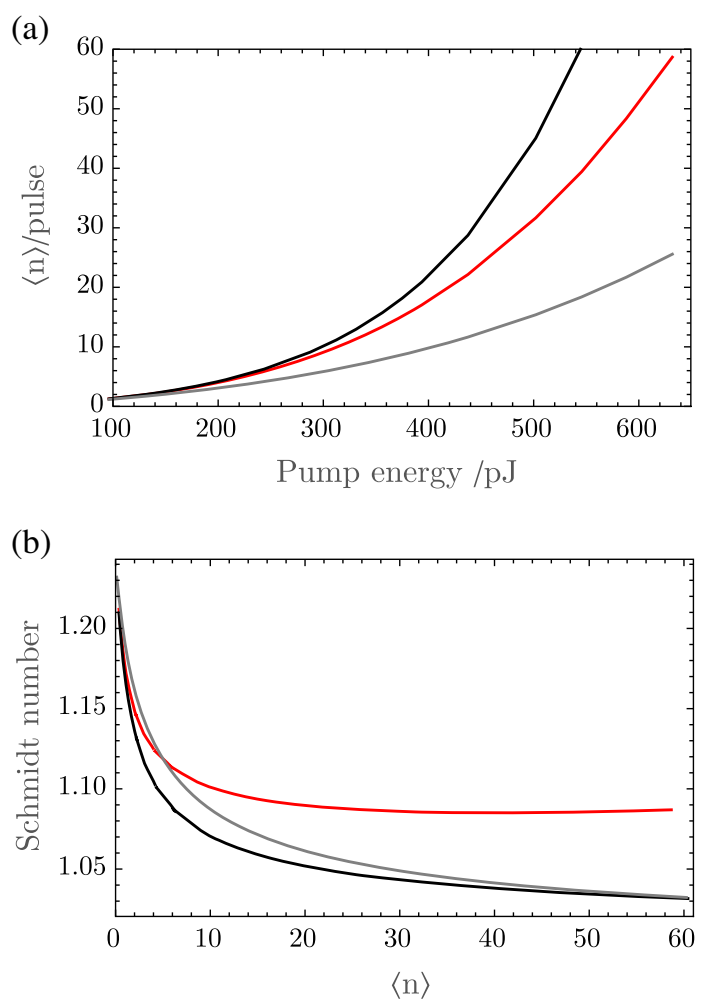

FIG. 8. (a) Number of spontaneously generated photons as a function of pump pulse energy. (b) Spectral Schmidt number as a function of the number of spontaneously generated photons. The red line shows the $\chi^{(2)} / \chi^{(3)}$ model; the black line is for the $\chi^{(2)}$ model (only PDC, no SPM or XPM); and the gray line is the simplified $\chi^{(2)}$ model (squeezing parameters $r_{l}=\sqrt{E_{\mathrm{p}}} c_{l}$ ) with the same predictions in the low-gain regime. Note that the curves converge in the low-gain regime but scale differently for higher gain.

More generally, the Schmidt number quantifies the maximum visibility of the second-order interference between the generated light and another optical mode [40]. It has been shown that the Schmidt number of a PDC source is reduced in the high-gain regime, due to the dominance of strongly populated modes [15,41]. We find that the pump SPM counteracts this effect: The Schmidt number saturates at a moderate pump power. Figure 8 illustrates these observations. Our simulations show that the effect of XPM on the spectral Schmidt number is negligible.

\section{Improving source performance}

We have shown that SPM due to the $\chi^{(3)}$ nonlinearity of KTP has an impact on the performance of our source in the high-gain regime. The results of our study, however, suggest a simple optimization to counteract this effect: prechirping of the pump pulses. If the sign and magnitude of the chirp are appropriately chosen, it is possible to counter the reduction in purity and brightness. In Fig. 9, we show the Schmidt number predicted by our theory as a function of the spontaneously generated photon number, 


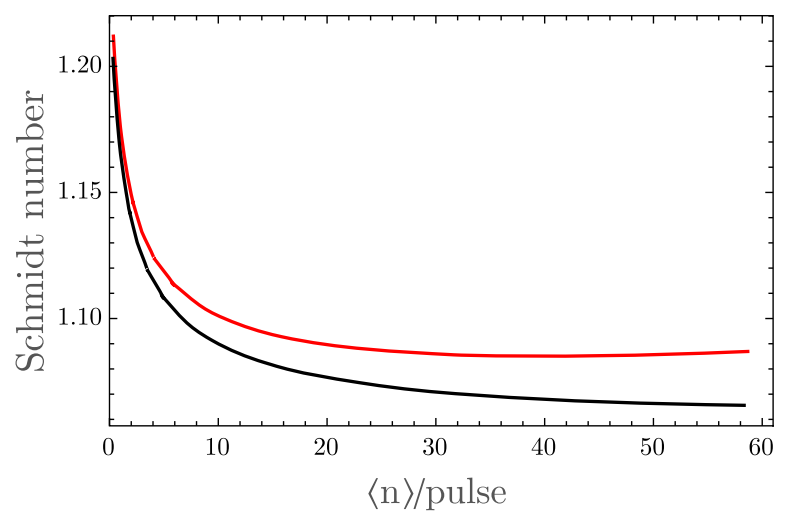

FIG. 9. Effect of prechirping pump pulses on the spectral Schmidt number of a PDC source as a function of the average number of photons generated. The red line is for the Fourierlimited pump pulses, and the black line is for the prechirped pump pulses.

contrasted with what could be achieved using chirped pump pulses. The dispersion parameter required to optimize the performance of the source is $D=178 \mathrm{fs} / \mathrm{nm}$, which can be easily achieved using a grating-based pulse compressor.

\section{CONCLUSIONS}

In this work, we have demonstrated a general experimental framework to characterize broadband twin-beam sources in the high-gain regime. We have introduced cascaded stimulated emission tomography, a seeded measurement that generalizes SET, providing additional spectral information about the generated twin beams. In particular, we have shown that the redundancy offered by this measurement allows for the self-referenced inference of the PDC interaction strength, independently of seeding and detection efficiency. Using the information offered by cascaded SET, together with a small number of complementary measurements, we have fitted a theoretical model of twin-beam generation, which yields all the complex TFs that describe the process. We have experimentally identified and quantified nonperturbative, SPM, and XPM effects in a high-gain PDC source. In fact, the development of the EOMs (6a), (6b), and (7) was prompted by features of the data that we were not initially able to explain. Our results also clarify the absence of observed deviations from a simplified $\chi^{(2)}$ model in previous work [4], as they can be effectively hidden by third-order phenomena. This completes the picture of using classical probes to tomograph twin-beam sources.

The twin-beam source setup that we used to develop and validate our methods is an example of a class of pulsed twin-beam sources engineered to suppress the emission of light in multiple spatial and spectral optical modes, such that the quantum states that they generate are useful for quantum simulation and quantum sampling applications
[3,6-11]. Our ability to modify the parameters of the model has allowed us to identify limitations of the current source design and to explore new designs that overcome these shortcomings. An interesting extension of our methods would include spatially multimode twin-beam sources. Efficient seeding of such sources has been demonstrated [42], and spatial input-output relations, as well as spatiotemporal correlations for parametric sources, have recently been discussed $[17,43]$.

We believe the framework presented here will advance the study and design of twin-beam sources in the high-gain regime. Comprehensive modeling and characterization based on the methods that we introduce can also be applied to many other important applications. Some examples are heralded Fock state sources for linear optical quantum computing and single-photon frequency conversion, both key requirements for many quantum technologies [23].

\section{ACKNOWLEDGMENTS}

G. T. thanks Merton College, Oxford, for its support. M. D. V. thanks the Engineering and Physical Sciences Research Council for funding through Grant No. EP/ K034480/1 (BLOQS). N.Q. and J.E.S. thank the National Science and Engineering Research Council of Canada. W. S. K. and I. A. W. thank EPSRC for Grant No. EP/T001062/1.

\section{APPENDIX A: QUANTUM AND CLASSICAL INPUT-OUTPUT RELATIONS}

We can find the exact transformation that describes the Heisenberg picture evolution of the creation or annihilation operators by looking at how it transforms the classical coherent amplitudes of a seed field. A broadband coherent seed in the signal (idler) mode can be described as a displaced vacuum, which in the Heisenberg picture can be represented by splitting a creation operation in terms of its mean value $\alpha_{\mathrm{s}, \mathrm{i}}$ and its fluctuations $\delta a_{\mathrm{s}, \mathrm{i}}$,

$$
a_{\mathrm{s}(\mathrm{i})}^{(\mathrm{in})}(\omega)=\alpha_{\mathrm{s}(\mathrm{i})}(\omega)+\delta a_{\mathrm{s}(\mathrm{i})}(\omega)
$$

The seed field is evolved following Eq. (1):

$$
\begin{aligned}
a_{\mathrm{s}}^{(\mathrm{out})}(\omega)= & \int d \omega^{\prime} U^{\mathrm{s}, \mathrm{s}}\left(\omega, \omega^{\prime}\right)\left[\delta a_{\mathrm{s}}\left(\omega^{\prime}\right)+\alpha_{s}\left(\omega^{\prime}\right)\right] \\
& +\int d \omega^{\prime} U^{\mathrm{s}, \mathrm{i}}\left(\omega, \omega^{\prime}\right)\left[\delta a_{\mathrm{i}}^{\dagger}\left(\omega^{\prime}\right)+\alpha_{i}\left(\omega^{\prime}\right)\right] .
\end{aligned}
$$

A spectrally resolved power measurement of the signal fields yields 


$$
\begin{aligned}
P_{s}(\omega) \propto\left\langle\left[a_{s}^{(\mathrm{out})}(\omega)\right]^{\dagger} a_{s}^{(\mathrm{out})}(\omega)\right\rangle \\
=\left|\int d \omega d \omega^{\prime} U^{\mathrm{s}, \mathrm{s}}\left(\omega, \omega^{\prime}\right) \alpha_{\mathrm{s}}\left(\omega^{\prime}\right)\right|^{2} \\
+\left|\int d \omega d \omega^{\prime} U^{\mathrm{s}, \mathrm{i}}\left(\omega, \omega^{\prime}\right) \alpha_{\mathrm{i}}\left(\omega^{\prime}\right)\right|^{2} \\
\quad+\left|\int d \omega d \omega^{\prime} U^{\mathrm{s}, \mathrm{i}}\left(\omega, \omega^{\prime}\right)\right|^{2},
\end{aligned}
$$

and a similar equation for the idler power. We identify three contributions to the PSD: The first, involving the self-mode TFs, represents the seed power and cascaded DFG. The second, involving the cross-mode $\mathrm{TF}$, represents stimulated emission (and higher-order cascaded DFG terms). The third term represents spontaneous emission. This last term is negligible for strong enough seed fields.

\section{APPENDIX B: SECOND-ORDER PERTURBATIVE EXPANSION OF PDC}

Here, we perturbatively solve the PDC equations of motion (6a) and (6b) up to second order in the PDC gain. We show that, while the term linear in $\gamma_{\mathrm{PDC}}$ yields the usual joint spectral amplitude, the second-order term yields a broadband pedestal in the same-mode transfer function. This case illustrates our simple physical picture: The same-mode emission is caused by a cascaded process where a broadband signal (idler) photon stimulated by the narrow-band idler (signal) seed subsequently stimulates broadband emission in the idler (signal) mode. Our fundamental integral equations are

$$
\begin{aligned}
& a_{\mathrm{S}}(z, \omega)=a_{\mathrm{s}}\left(z_{0}, \omega\right) e^{i \Delta k_{\mathrm{s}}(\omega)\left(z-z_{0}\right)}+\frac{i \gamma_{\mathrm{PDC}}}{\sqrt{2 \pi}} \int_{z_{0}}^{z} d z^{\prime} g\left(z^{\prime}\right) e^{i \Delta k_{\mathrm{s}}(\omega)\left(z-z^{\prime}\right)} \int d \omega^{\prime} \beta_{\mathrm{p}}\left(z^{\prime}, \omega+\omega^{\prime}\right) a_{\mathrm{i}}^{\dagger}\left(z^{\prime}, \omega^{\prime}\right), \\
& a_{\mathrm{i}}(z, \omega)=a_{\mathrm{i}}\left(z_{0}, \omega\right) e^{i \Delta k_{\mathrm{i}}(\omega)\left(z-z_{0}\right)}+\frac{i \gamma_{\mathrm{PDC}}}{\sqrt{2 \pi}} \int_{z_{0}}^{z} d z^{\prime} g\left(z^{\prime}\right) e^{i \Delta k_{\mathrm{i}}(\omega)\left(z-z^{\prime}\right)} \int d \omega^{\prime} \beta_{\mathrm{p}}\left(z^{\prime}, \omega+\omega^{\prime}\right) a_{\mathrm{s}}^{\dagger}\left(z^{\prime}, \omega^{\prime}\right) .
\end{aligned}
$$

The equations above are clearly formal solutions of Eqs. (6a) and (6b). Taking the adjoints and substituting into Eq. (B1), we have

$$
\begin{aligned}
a_{\mathrm{s}}(z, \omega)= & a_{\mathrm{s}}\left(z_{0}, \omega\right) e^{i \Delta k_{\mathrm{s}}(\omega)\left(z-z_{0}\right)}+\frac{i \gamma_{\mathrm{PDC}}}{\sqrt{2 \pi}} \int_{z_{0}}^{z} d z^{\prime} g\left(z^{\prime}\right) e^{i \Delta k_{\mathrm{s}}(\omega)\left(z-z^{\prime}\right)} \int d \omega^{\prime} \beta_{\mathrm{p}}\left(z^{\prime}, \omega+\omega^{\prime}\right) e^{-i \Delta k_{\mathrm{i}}\left(\omega^{\prime}\right)\left(z^{\prime}-z_{0}\right)} a_{\mathrm{i}}^{\dagger}\left(z_{0}, \omega^{\prime}\right) \\
& +\frac{\gamma_{\mathrm{PDC}}^{2}}{2 \pi} \int_{z_{0}}^{z} d z^{\prime} g\left(z^{\prime}\right) \int_{z_{0}}^{z^{\prime}} d z^{\prime \prime} g^{*}\left(z^{\prime \prime}\right) e^{i \Delta k_{\mathrm{s}}(\omega)\left(z-z^{\prime}\right)} \iint d \omega^{\prime} d \omega^{\prime \prime} \beta_{\mathrm{p}}\left(z^{\prime}, \omega+\omega^{\prime}\right) e^{-i \Delta k_{\mathrm{i}}\left(\omega^{\prime}\right)\left(z^{\prime}-z^{\prime \prime}\right)} \beta_{\mathrm{p}}^{*}\left(z^{\prime \prime}, \omega^{\prime}+\omega^{\prime \prime}\right) a_{\mathrm{s}}\left(z^{\prime \prime}, \omega^{\prime \prime}\right),
\end{aligned}
$$

and a similar equation for the evolution of $a_{\mathrm{i}}(z, \omega)$ obtained by letting $\mathrm{s} \leftrightarrow \mathrm{i}$ in the last equation. In what follows, we only write equations for the signal, but it is understood that a similar equation follows for the idler using the rule written in the last sentence.

These expressions are still exact. Beginning an iteration and looking at a final $z=z_{1}$, we have

$$
\begin{aligned}
a_{\mathrm{s}}\left(z_{1}, \omega\right)= & a_{\mathrm{s}}\left(z_{0}, \omega\right) e^{i \Delta k_{\mathrm{s}}(\omega)\left(z_{1}-z_{0}\right)}+\frac{i \gamma_{\mathrm{PDC}}}{\sqrt{2 \pi}} \int_{z_{0}}^{z_{1}} d z^{\prime} g\left(z^{\prime}\right) e^{i \Delta k_{\mathrm{s}}(\omega)\left(z_{1}-z^{\prime}\right)} \int d \omega^{\prime} \beta_{\mathrm{p}}\left(z^{\prime}, \omega+\omega^{\prime}\right) e^{-i \Delta k_{\mathrm{i}}\left(\omega^{\prime}\right)\left(z^{\prime}-z_{0}\right)} a_{\mathrm{i}}^{\dagger}\left(z_{0}, \omega^{\prime}\right) \\
& +\frac{\gamma_{\mathrm{PDC}}^{2}}{2 \pi} \int_{z_{0}}^{z_{1}} d z^{\prime} g\left(z^{\prime}\right) \int_{z_{0}}^{z^{\prime}} d z^{\prime \prime} g^{*}\left(z^{\prime \prime}\right) e^{i \Delta k_{\mathrm{s}}(\omega)\left(z_{1}-z^{\prime}\right)} \\
& \times \iint d \omega^{\prime} d \omega^{\prime \prime} \beta_{\mathrm{p}}\left(z^{\prime}, \omega+\omega^{\prime}\right) e^{-i \Delta k_{\mathrm{i}}\left(\omega^{\prime}\right)\left(z^{\prime}-z^{\prime \prime}\right)} \beta_{\mathrm{p}}^{*}\left(z^{\prime \prime}, \omega^{\prime}+\omega^{\prime \prime}\right) e^{i \Delta k_{\mathrm{s}}\left(\omega^{\prime \prime}\right)\left(z^{\prime \prime}-z_{0}\right)} a_{\mathrm{s}}\left(z_{0}, \omega^{\prime \prime}\right) .
\end{aligned}
$$

Then, writing

$$
\begin{aligned}
& a_{\mathrm{s}}^{(\text {out })}(\omega)=\int d \omega^{\prime \prime} U^{s, s}\left(\omega, \omega^{\prime \prime}\right) a_{\mathrm{s}}^{(\text {in })}\left(\omega^{\prime \prime}\right)+\int d \omega^{\prime} U^{s, i}\left(\omega, \omega^{\prime}\right) a_{\mathrm{i}}^{\dagger(\text { in })}\left(\omega^{\prime}\right), \\
& a_{\mathrm{i}}^{\text {(out) }}(\omega)=\int d \omega^{\prime \prime} U^{i, i}\left(\omega, \omega^{\prime \prime}\right) a_{\mathrm{i}}^{(\text {in })}\left(\omega^{\prime \prime}\right)+\int d \omega^{\prime} U^{i, s}\left(\omega, \omega^{\prime}\right) a_{\mathrm{s}}^{\dagger(\text { in })}\left(\omega^{\prime}\right), \\
& a_{\mathrm{x}}^{(\text {in })}(\omega)=e^{-i \Delta k_{\mathrm{x}}(\omega) z_{0}} a_{\mathrm{x}}\left(z_{0}, \omega\right), \quad a_{\mathrm{x}}^{(\text {out })}(\omega)=e^{-i \Delta k_{\mathrm{x}}(\omega) z_{1}} a_{\mathrm{x}}\left(z_{1}, \omega\right),
\end{aligned}
$$

we have 


$$
\begin{aligned}
U^{\mathrm{s}, \mathrm{s}}\left(\omega, \omega^{\prime \prime}\right)= & \delta\left(\omega-\omega^{\prime \prime}\right) \\
& +\frac{\gamma_{\mathrm{PDC}}^{2}}{2 \pi} \int_{z_{0}}^{z_{1}} d z^{\prime} g\left(z^{\prime}\right) \int_{z_{0}}^{z^{\prime}} d z^{\prime \prime} g^{*}\left(z^{\prime \prime}\right) e^{-i \Delta k_{\mathrm{s}}(\omega) z^{\prime}} \int d \omega^{\prime} \beta_{\mathrm{p}}\left(z^{\prime}, \omega+\omega^{\prime}\right) e^{-i \Delta k_{\mathrm{i}}\left(\omega^{\prime}\right)\left(z^{\prime}-z^{\prime \prime}\right)} \beta_{\mathrm{p}}^{*}\left(z^{\prime \prime}, \omega^{\prime \prime}+\omega^{\prime}\right) e^{i \Delta k_{\mathrm{s}}\left(\omega^{\prime \prime}\right) z^{\prime \prime}} \\
U^{\mathrm{s}, \mathrm{i}}\left(\omega, \omega^{\prime}\right)= & \frac{i \gamma_{\mathrm{PDC}}}{\sqrt{2 \pi}} \int_{z_{0}}^{z_{1}} d z^{\prime} e^{-i \Delta k_{\mathrm{s}}(\omega) z^{\prime}} g\left(z^{\prime}\right) \beta_{\mathrm{p}}\left(z^{\prime}, \omega+\omega^{\prime}\right) e^{-i \Delta k_{\mathrm{i}}\left(\omega^{\prime}\right) z^{\prime}}
\end{aligned}
$$

The first-order perturbative result in $U^{\mathrm{s}, \mathrm{i}}$ in the last set of equations describes how a field in the idler (signal) mode stimulates the generation of a field in the signal (idler) mode, and it is often employed in the context of photo-pair generation, where the first-order approximation of $U^{\mathrm{s}, \mathrm{i}(\mathrm{i}, \mathrm{s})}\left(\omega, \omega^{\prime}\right)$ is the joint spectrum of SPDC photon pairs. This set of equations can be written in a more elegant form by introducing the phase-matching function (PMF)

$$
\begin{gathered}
\Phi\left[\Delta k\left(\omega, \omega^{\prime}\right)\right]=\int_{z_{0}}^{z_{1}} \frac{d z}{\sqrt{2 \pi}} g(z) e^{-i \Delta k\left(\omega, \omega^{\prime}\right) z}, \\
\Delta k\left(\omega, \omega^{\prime}\right)=\Delta k_{\mathrm{s}}(\omega)+\Delta k_{\mathrm{i}}\left(\omega^{\prime}\right) \\
=\left(\Delta \beta_{\mathrm{s}}\right)\left(\omega-\bar{\omega}_{\mathrm{s}}\right)+\left(\Delta \beta_{\mathrm{i}}\right)\left(\omega^{\prime}-\bar{\omega}_{\mathrm{i}}\right), \\
\Delta \beta_{x}=1 / v_{x}-1 / v_{\mathrm{p}},
\end{gathered}
$$

and recalling that, in the low-gain regime, the spectral content of the pump is not changed, allowing us to write

$$
\beta_{\mathrm{p}}\left(z, \omega+\omega^{\prime}\right)=\sqrt{\frac{E_{\mathrm{p}}}{\sigma}} F\left(\frac{\omega-\bar{\omega}_{\mathrm{s}}+\omega^{\prime}-\bar{\omega}_{\mathrm{i}}}{\sigma}\right),
$$

where $F(x)$ is the $\mathcal{L}^{2}$ normalized pump shape [i.e., $\left.\int d x|F(x)|^{2}=1\right]$ and we used the fact that $\bar{\omega}_{\mathrm{p}}=\bar{\omega}_{\mathrm{s}}+\bar{\omega}_{\mathrm{i}}$. With these definitions, we finally write

$U^{\mathrm{s}, \mathrm{i}}\left(\omega, \omega^{\prime}\right)=i \gamma_{\mathrm{PDC}} \sqrt{\frac{E_{\mathrm{p}}}{\sigma}} F\left(\frac{\omega+\omega^{\prime}-\bar{\omega}_{\mathrm{p}}}{\sigma}\right) \Phi\left(\Delta k\left(\omega, \omega^{\prime}\right)\right)$.

Note that if, for example, $g(z)$ is a top-hat function of length $L$ centered at the origin, then

$$
\Phi\left(\Delta k\left(\omega, \omega^{\prime}\right)\right)=\frac{L}{\sqrt{2 \pi}} \operatorname{sinc}\left(\Delta k\left(\omega, \omega^{\prime}\right) L / 2\right)
$$

The mean number of spontaneously generated signal (idler) photons per pump pulse is thus

$$
\begin{aligned}
\left\langle n_{\mathrm{s}(\mathrm{i})}\right\rangle & =\int d \omega\left\langle a_{\mathrm{s}}^{\dagger}(\omega) a_{\mathrm{s}}(\omega)\right\rangle=\int d \omega d \omega^{\prime}\left|U^{\mathrm{s}, \mathrm{i}}\left(\omega, \omega^{\prime}\right)\right|^{2} \\
& =\frac{\gamma_{\mathrm{PDC}}^{2} E_{\mathrm{p}} \ell}{\left|\frac{1}{v_{\mathrm{s}}}-\frac{1}{v_{\mathrm{i}}}\right|}
\end{aligned}
$$

where $\ell=\int_{\ell_{\min }}^{\ell_{\max }} d z|g(z)|^{2}$ is the effective length of the crystal. Note that a $g(z)$ with a top-hat shape of length $L$ will have precisely $\ell=L$.

When the idler(signal) mode is seeded, broadband DFG will be generated in the conjugate mode (Appendix A) with a total intensity $I_{\mathrm{S}(\mathrm{i})} \propto$ $\int d \omega d \omega^{\prime}\left|U^{\mathrm{s}, \mathrm{i}(\mathrm{i}, \mathrm{s})}\left(\omega, \omega^{\prime}\right)\right|^{2}=\left\langle n_{\mathrm{s}(\mathrm{i})}\right\rangle$.

The second-order term that appears in $U^{\mathrm{s}, \mathrm{s}}\left(\omega, \omega^{\prime}\right)$ and $U^{\mathrm{i}, \mathrm{i}}\left(\omega, \omega^{\prime}\right)$ generates a broadband contribution in the seeded mode with amplitude proportional to the square of the PDC gain.

Under certain circumstances, it is possible to evaluate this term analytically. For this evaluation, we assume a Gaussian nonlinearity profile and pump profile,

$$
g(z)=\frac{1}{\sqrt[4]{\pi \gamma / 2}} e^{-\frac{z^{2}}{\gamma \ell^{2}}}, \quad F(x)=\frac{1}{\sqrt[4]{\pi}} e^{-\frac{x^{2}}{2}},
$$

where $\gamma \approx 0.193$ is chosen so that the PMF associated with this nonlinearity profile has the same full width at half maximum as the one associated with a top-hat profile. Under these approximations, letting $z_{0} \rightarrow-\infty$ and $z_{1} \rightarrow+\infty$, one can write the intermode transfer function as a double Gaussian,

$$
\begin{gathered}
U^{\mathrm{s}, \mathrm{i}}\left(\omega, \omega^{\prime}\right)=i \gamma_{\mathrm{PDC}} \ell \sqrt{\frac{E_{\mathrm{p}}}{\pi \sigma} \sqrt{\gamma / 2}} \exp \left(-\mathbf{v M \mathbf { v } ^ { T }}\right), \\
\mathbf{v}=\left(\omega-\bar{\omega}_{\mathrm{s}}, \omega^{\prime}-\bar{\omega}_{\mathrm{i}}\right), \\
\mathbf{M}=\left(\begin{array}{cc}
\mu_{\mathrm{s}}^{2} & \mu^{2} \\
\mu^{2} & \mu_{\mathrm{i}}^{2}
\end{array}\right), \\
\mu_{\mathrm{s}, \mathrm{i}}^{2}=\frac{1}{4} \ell^{2} \gamma \Delta \beta_{\mathrm{s}, \mathrm{i}}^{2}+\frac{1}{2 \sigma^{2}}, \\
\mu^{2}=\frac{1}{4} \gamma \Delta \beta_{\mathrm{i}} \Delta \beta_{\mathrm{s}} \ell^{2}+\frac{1}{2 \sigma^{2}} .
\end{gathered}
$$


Similarly, one can write a simple analytical expression for the second-order intramode transfer function,

$$
\begin{aligned}
U^{\mathrm{s}, \mathrm{s}}\left(\omega, \omega^{\prime \prime}\right) & =\delta\left(\omega-\omega^{\prime \prime}\right)+U_{\mathrm{b}}^{\mathrm{s}, \mathrm{s}}\left(\omega, \omega^{\prime \prime}\right), \\
U_{\mathrm{b}}^{\mathrm{s}, \mathrm{s}}\left(\omega, \omega^{\prime \prime}\right) & =\frac{\gamma_{\mathrm{PDC}}^{2} E_{\mathrm{p}} \ell^{2} \sqrt{\gamma}}{\sqrt{\pi} \sigma \mu_{\mathrm{i}}} e^{-x_{-}^{2}-x_{+}^{2}}\left(1+i \operatorname{erfi}\left(x_{+}\right)\right), \\
x_{+} & =\frac{\frac{\sqrt{\gamma} \ell}{2}\left(\frac{1}{v_{\mathrm{s}}}-\frac{1}{v_{\mathrm{i}}}\right)}{2 \sigma \mu_{\mathrm{i}}}\left(\omega+\omega^{\prime \prime}-2 \bar{\omega}_{\mathrm{s}}\right), \\
x_{-} & =\frac{\mu_{\mathrm{s}}}{\sqrt{2}}\left(\omega-\omega^{\prime \prime}\right),
\end{aligned}
$$

and $\operatorname{erfi}(x)=\operatorname{erf}(i x) / i$ is the imaginary error function, which is an odd function of its argument. Thus, the phase of $U_{\mathrm{b}}^{\mathrm{s}, \mathrm{s}}\left(\omega, \omega^{\prime \prime}\right)$ has a jump when crossing the line $\omega+\omega^{\prime \prime}-2 \bar{\omega}_{\mathrm{s}}=0$. This observation will become useful when we compare the form of the transfer function of the cascaded (second-order) $\chi^{(2)}$ process, which, as just mentioned, is a phase jump, and the pure $\chi^{(3)}$ process, which does not. Finally, note that an analogous expression for the intramode transfer function $U_{\mathrm{b}}^{i, \mathrm{i}}\left(\omega, \omega^{\prime \prime}\right)$ can be obtained by letting $\mathrm{i} \leftrightarrow \mathrm{s}$ in Eq. (B21).

\section{APPENDIX C: EXPERIMENTAL SETUP DETAILS}

The nonlinear crystal in our experiment is an 8-mm PPKTP chip manufactured by ADVR, made from a $z$-cut wafer, with a set of waveguides written using a proton exchange process. The waveguides have roughly $4 \mu \mathrm{m} \times$ $4 \mu \mathrm{m}$ cross sections. The chip output is AR coated for $1550 \mathrm{~nm}$. We pump the source with a pulsed Ti:Sapphire laser (Coherent Chameleon) with central wavelength $783 \mathrm{~nm}, 8-\mathrm{nm}$ bandwidth, and $80-\mathrm{MHz}$ repetition rate, which we filter using two bandpass thin-film filters (Semrock LL01-785-12.5) to a bandwidth of $1.9 \mathrm{~nm}$. The periodic poling has a spatial period of $104 \mu \mathrm{m}$, which leads to quasi-phase matching for a signal and an idler around $1560 \mathrm{~nm}$. Using an ordinarily polarized pump ( $\mathrm{H}$ polarization), we obtain signal $(\mathrm{H}$ polarization) and idler (V polarization) beams with respective central wavelengths of $1563 \mathrm{~nm}$ and $1569 \mathrm{~nm}$.

The phase matching of the type-II PDC process has been engineered to obtain signal and idler fields at telecom wavelengths with an almost separable joint spectrum. In the weak squeezing regime, where we may approximate that only single-photon pairs are produced, the joint spectrum of a PDC source can be expressed as a product of two factors: a pump function and a phase-matching function [35]. As illustrated in Fig. 10, the pump function, related to energy conservation, introduces anticorrelation of the frequencies
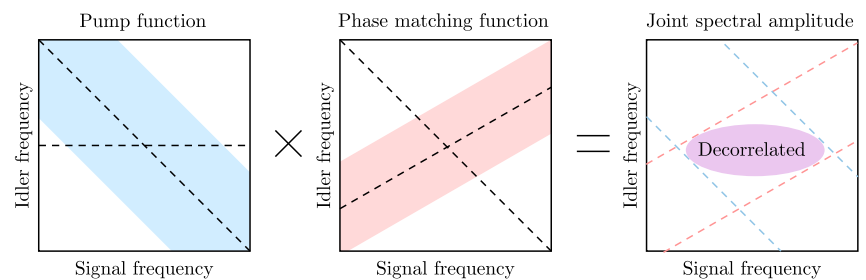

FIG. 10. The product of a pump function (energy conservation) and a phase-matching function (momentum conservation) can yield a separable joint spectrum.

of photons generated in the PDC process. The nature of the phase-matching function, related to momentum conservation, depends on the dispersion relation of the guided modes. In a medium with normal dispersion, both the signal and idler commonly have higher group velocity than the pump, resulting in frequency anticorrelations [44]. In the waveguided PPKTP, due to the birefringence of the material, the group velocity of a pump field at $783 \mathrm{~nm}$ in the ordinary polarization falls in between the group velocities of the signal and idler fields centered around $1565 \mathrm{~nm}$ in orthogonal polarizations, which results in a phase-matching function with positive frequency correlations [2]. The combined effect of these two factors with the correct balance of pump and phase-matching bandwidths, as illustrated in Fig. 10, can produce an almost separable joint spectrum.

To measure the direct and cascaded DFG signals, we seed one of the down-converted fields with a cw laser and measure the generated stimulated emission using an OSA. For the seed field, we use a Yenista Tunics T100S-HP cw fiber laser with a wavelength tunable between $1500 \mathrm{~nm}$ and $1680 \mathrm{~nm}$. The laser has a very high spontaneous noise suppression, over $100 \mathrm{~dB}$ over a bandwidth of $1 \mathrm{~nm}$. After polarization filtering using birefringent waveplates and a Glan-Taylor polarizer, we record spectra of the generated light using a Yokogawa AQ6370D OSA. The OSA has a high dynamic range of $78 \mathrm{~dB}$ over $1 \mathrm{~nm}$, allowing us to distinguish the weak broadband cascaded DFG signal $(<1 \mathrm{nW})$ from the narrow-band seed $(\approx 600 \mu \mathrm{W})$. While the linewidth of the seed laser is many orders of magnitude smaller than the broadband cascaded DFG emission, the finite resolution of the OSA $(0.2 \mathrm{~nm})$ broadens the width of the narrow-band component of the spectra, hindering the retrieval of the broadband pedestal. To remove the $\mathrm{cw}$ seed from the data, we first subtract a background trace from it, where we send the seed beam but not the pump through the crystal. Fluctuations of the seed power between the moment when the data and the background are measured led to an imperfect extinction of the narrow-band component. We eliminate this $\mathrm{cw}$ remnant by masking the frequency range occupied by it and interpolating the remaining spectrum, as illustrated in Fig. 11. 


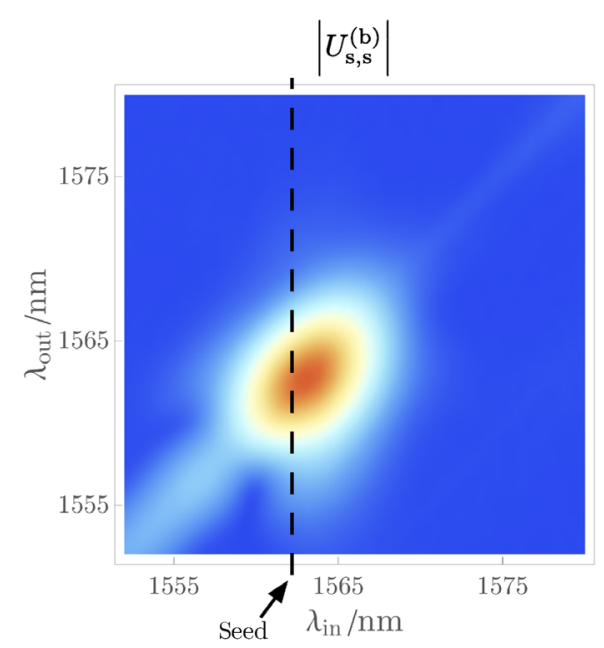

Output spectrum

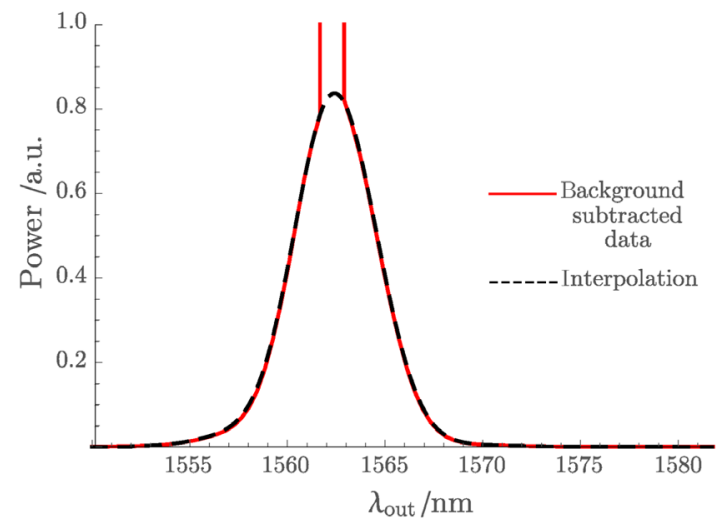

FIG. 11. (a) Reconstruction of the stimulated emission spectrum as a function of seeding wavelength, measured in the seed polarization. Color indicates the absolute value of the field (square root of the measured spectral power density). (b) The red line represents one "slice" of the background subtracted data, showing a noisy measurement around the seed wavelength, where the OSA detector is blinded by the seed; the black line is the reconstructed spectrum obtained by interpolating over the noisy region.

\section{APPENDIX D: IDENTIFYING THE EOM PARAMETERS}

In this Appendix, we describe in detail how we identify the physical parameters that enter in our theoretical description in Eqs. (6a), (6b), and (7) of the SPDC. We describe how these parameters can be extracted from the TF data mostly in the small-gain limit, complemented with some additional measurements.

\section{Pump spectral intensity}

The pump spectral amplitude at position $z$ in the crystal, $\beta_{\mathrm{p}}(z, \omega)$, is a complex-valued function. In our experiment, we measure the absolute value of $\beta_{\mathrm{p}}(z=0, \omega)$ in two ways (see Fig. 12): First, we perform a direct measurement of the

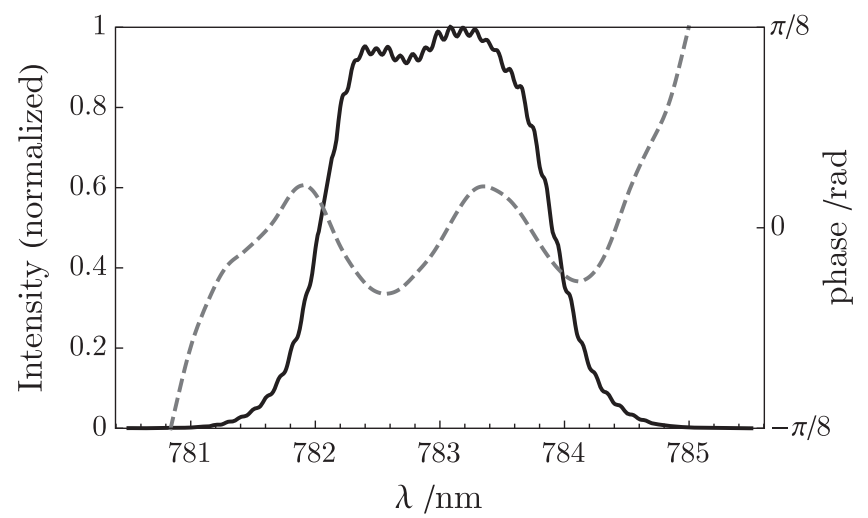

FIG. 12. Magnitude (solid line) and phase (dashed line) of the filtered pump spectral amplitude. The magnitude shown here corresponds to that measured using the OSA and agrees very well with the pump function of a low-gain JSI. The phase shown here corresponds to the addition of the phase of the unfiltered pump measured using SPIDER and the differential phase of the filtered pump measured through spectral interferometry.

pump spectrum at the chip input using an OSA, which provides the squared modulus of the pump spectral amplitude. Second, we confirm this result by extracting the pump spectral density from low-gain cross-mode TF measurements.

\section{Pump spectral phase}

We used an APE LX-Spider (wavelength range between 750 and $900 \mathrm{~nm}$ ) to characterize the spectral phase of the Ti:Sapphire pump laser, but we could not extend that measurement to the filtered pump (1.9-nm bandwidth) because of the limit in the spectral resolution of the apparatus. We characterized the spectral phase added by the filters using a spectral self-interference measurement: The filtered pump is interfered with a sample of the same, unfiltered field, thus revealing their differential spectral phase. The total spectral phase of the pump is obtained by adding the spectral phase of the reference beam, measured using SPIDER, and the phase added by the optical filters, measured through spectral interference.

Let us briefly detail the spectral interference process by which we measure the phase added by the optical filters. The complex spectral amplitude corresponding to the sum of the unfiltered reference $\beta_{\text {ref }}$ and the filtered pump field $\beta_{\mathrm{p}}(\omega)$ is

$$
\beta_{\text {sum }}(\omega)=\beta_{\text {ref }}+\left|\beta_{\mathrm{p}}(\omega)\right| \exp [i(\phi(\omega)+\Delta t \omega)],
$$

where we omit the spatial label in the field amplitudes, implicitly assuming a position before the chip input. We assume that the unfiltered reference is much broader than the filtered pump, such that the former can be treated as a field with a constant spectral density. Without loss of generality, we also take the spectral phase as constant. 
The phase of the filtered pump is the sum of $\phi(\omega)$, the phase added in the filtering process, and $\Delta t \omega$, representing a temporal delay with respect to the reference field.

The measured PSD is

$$
\begin{aligned}
P(\omega) & \propto\left|\beta_{\text {sum }}(\omega)\right|^{2} \\
& =\left|\beta_{\text {ref }}\right|^{2}+\left|\beta_{\mathrm{p}}(\omega)\right|^{2}+\left|\beta_{\text {ref }}\right|\left|\beta_{\mathrm{p}}(\omega)\right| \cos (\phi(\omega)-\Delta t \omega),
\end{aligned}
$$

where we recognize that the last term describes spectral fringes with a period of approximately $1 / \Delta t$, modulated by the spectral phase added by the optical filters. We assume that the delay between the interfering beams is large, so the interference fringes that it causes are fast compared with the spectral interference due to the filters' spectral phase. This modulation can be retrieved by keeping only positive frequencies above the bandwidth of the base-band component of $I(\omega)$, which corresponds to the complex signal

$$
P_{\mathrm{HF}} \propto\left|\beta_{\mathrm{ref}}\right|\left|\beta_{\mathrm{p}}(\omega)\right| \exp [\Delta t \omega+\phi(\omega)]
$$

the argument of which corresponds to the spectral phase added by the filters, modulo a linear component.

Our SPIDER measurement reveals that the chirp introduced in the unfiltered pump by the power-distribution optics is small, reaching less than $0.1 \mathrm{rad}$ over the filtered pump bandwidth. The spectral phase added by the dielectric filters is the main contribution to the total spectral phase. This measurement agrees with the specifications provided by the supplier of the filters (Semrock). In Fig. 12, we show the measured magnitude and phase of the pump spectral amplitude.

\section{Group-velocity mismatch}

In our model, the group velocities of the pump, signal, and idler fields determine the linear propagation of the fields in the crystal. We need the two group-velocity mismatch parameters, $\Delta \beta_{\mathrm{s}(\mathrm{i})}=1 / v_{\mathrm{s}(\mathrm{i})}-1 / v_{\mathrm{p}}$. The phasematching (PM) function (see Appendix B) of a low-gain joint spectral amplitude has the simple form

$$
\Phi_{\mathrm{PM}}\left(\Omega_{\mathrm{s}}, \Omega_{\mathrm{i}}\right) \propto \operatorname{sinc}\left(\frac{L}{2}\left(\Delta \beta_{\mathrm{s}} \Omega_{\mathrm{s}}+\Delta \beta_{\mathrm{i}} \Omega_{\mathrm{i}}\right)\right),
$$

where $L$ is the crystal length and $\Omega_{\mathrm{s}(\mathrm{i})}=\omega_{\mathrm{s}(\mathrm{i})}-\bar{\omega}_{\mathrm{s}(\mathrm{i})}$ are the detunings from the central phase-matching frequencies. Perfect phase-matching occurs for frequencies $\Omega_{\mathrm{i}}=$ $\Omega_{\mathrm{S}} \tan \left(\theta_{\mathrm{PM}}\right)$, with $\tan \left(\theta_{\mathrm{PM}}\right)=-\Delta \beta_{\mathrm{s}} / \Delta \beta_{\mathrm{i}}$. Rotating to a frequency frame by the phase-matching angle, $\theta_{\mathrm{PM}}$,

$$
\begin{aligned}
& \Omega_{\|}=\cos \left(\theta_{\mathrm{PM}}\right) \Omega_{\mathrm{s}}+\sin \left(\theta_{\mathrm{PM}}\right) \Omega_{\mathrm{i}}, \\
& \Omega_{\perp}=\cos \left(\theta_{\mathrm{PM}}\right) \Omega_{\mathrm{i}}-\sin \left(\theta_{\mathrm{PM}}\right) \Omega_{\mathrm{s}},
\end{aligned}
$$

the phase-matching function depends only on the perpendicular component:

$$
\Phi_{\mathrm{PM}}\left(\Omega_{\perp}\right) \propto \operatorname{sinc}\left(\frac{1}{2} \Omega_{\perp} \frac{\Delta \mu}{\cos \left(\theta_{\mathrm{PM}}\right)+\sin \left(\theta_{\mathrm{PM}}\right)}\right),
$$

where we have introduced the quantity $\Delta \mu=$ $L\left(\Delta \beta_{\mathrm{s}}-\Delta \beta_{\mathrm{i}}\right)=L\left(1 / v_{\mathrm{s}}-1 / v_{\mathrm{i}}\right)$, which corresponds to the group delay between the signal and idler over the crystal length. This parametrization makes it clear that, in the symmetric group-velocity-matching case, the phasematching bandwidth is inversely proportional to the walkoff between the signal and idler pulses in the crystal. We can write the group-velocity-mismatch parameters $\Delta \beta_{\mathrm{s}(\mathrm{i})}$ in terms of $\Delta \mu$ and $\theta_{\mathrm{PM}}$ as

$$
\begin{gathered}
\Delta \beta_{\mathrm{s}}=\frac{\Delta \mu}{L} \frac{\tan \left(\theta_{\mathrm{PM}}\right)}{\tan \left(\theta_{\mathrm{PM}}\right)+1}, \\
\Delta \beta_{\mathrm{i}}=-\frac{\Delta \mu}{L} \frac{1}{\tan \left(\theta_{\mathrm{PM}}\right)+1} .
\end{gathered}
$$

To obtain the phase-matching angle $\theta_{\mathrm{PM}}$, we reconstruct a broad section of the phase-matching function by measuring the cross-mode TF as we scan the pump power and by joining the resulting measurements [see Fig. 13(a)]. We find the phase-matching angle $\theta_{\mathrm{PM}}=59.2^{\circ}$. (a) Phase matching angle

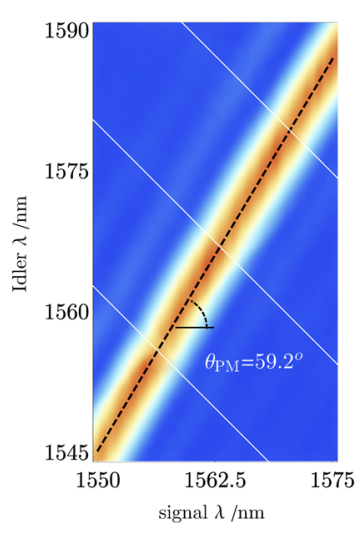

(b) Phase matching profile

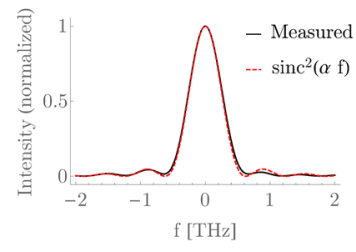

(c) Birefringent walk-off

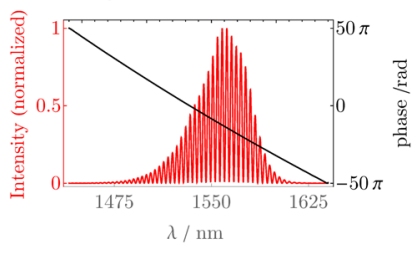

FIG. 13. Group-velocity-mismatch characterization. (a) Measurement of the phase-matching angle. Four low-gain JSIs with different pump central frequencies are joined to reconstruct a long section of the phase-matching function. (b) Inference of $\Delta \mu$ by measuring the bandwidth of the phase-matching profile. The measured intensity profile (solid black line) is fitted with a sinc ${ }^{2}$ function (dashed red line), and the group delay is obtained from Eq. (D7). (c) Direct measurement of $\Delta \mu$ by measuring spectral interference between two orthogonal polarizations of a broadband SLED after traveling through the crystal. The oscillating spectral intensity (red line) is transformed to the Fourier domain, and a corresponding spectral phase is obtained (black line), from which the spectral oscillation period is retrieved. 
The second parameter, $\Delta \mu$, can be obtained by measuring the spectral interference due to walk-off between pulses in the signal and idler polarizations propagating through the source. We launch broadband light from a very broadband $(\sim 100 \mathrm{~nm})$ superluminiscent light-emitting diode (SLED) in diagonal polarization through the PPKTP waveguide. The input polarization state can be written as $|D\rangle=$ $(|H\rangle+|V\rangle) / \sqrt{2}$. The output polarization state is then $\left(e^{i \phi_{\mathrm{H}}}|H\rangle+e^{i \phi_{\mathrm{V}}}|V\rangle\right) / \sqrt{2}$. Up to first order in the wavelength $\lambda$, we have

$$
\begin{aligned}
& \phi_{\mathrm{V}}-\phi_{\mathrm{H}} \\
& =\omega_{0} L\left[\left(\frac{n_{\mathrm{V}}\left(\omega_{0}\right)-n_{\mathrm{H}}\left(\omega_{0}\right)}{c}+\frac{1}{v_{\mathrm{i}}}-\frac{1}{v_{\mathrm{s}}}\right)+\left(\frac{1}{v_{\mathrm{s}}}-\frac{1}{v_{\mathrm{i}}}\right) \frac{\lambda}{\lambda_{0}}\right] \\
& =\text { constant }+\frac{2 \pi c}{\lambda_{0}^{2}} \Delta \mu \lambda,
\end{aligned}
$$

where $\omega_{0}$ is the central frequency of the signal or idler fields, $\lambda_{0}$ is the corresponding central wavelength in vacuum, and $n_{\mathrm{V} / \mathrm{H}}\left(\omega_{0}\right)$ is the refractive index experienced by the vertical/horizontal polarizations of a field at said central wavelength. Thus, when we project the output on the diagonal polarization, in the output wavelength spectrum we will observe fringes with period $\lambda_{0}^{2} /(c \Delta \mu)$. Figure 13(c) shows our measurement of these spectral fringes, from which we extract a delay between the signal and idler of $\Delta \mu=2.2 \mathrm{ps}$.

As shown in Fig. 13(b), we independently infer the value of $\Delta \mu$ by measuring the phase-matching profile of a lowgain JSI and fitting it to the function predicted in Eq. (D7). While it is known that inhomogeneities in the periodic poling of a nonlinear crystal lead to distortions of the phasematching profile $[45,46]$, the good fit of the data to a "sinc" function allows us to assume that the periodic poling in this waveguide does not show a significant inhomogeneity. We must note that this was not the case in other waveguides of the same chip, which yielded phase-matching profiles showing important deviations from a sinc function (see Fig. 14). The value of the group delay that fits the bandwidth of the measured phase-matching profile is again $\Delta \mu=2.2 \mathrm{ps}$, confirming our previous measurement.

The values of the group-velocity-mismatch parameters retrieved from Eqs. (D8a) and (D8b), together with the results of our measurements, and assuming a crystal of length $L=8 \mathrm{~mm}$, are $\beta_{s}=1.7 \mathrm{ps} / \mathrm{cm}$ and $\beta_{i}=-1.0 \mathrm{ps} / \mathrm{cm}$.

\section{Parametric down-conversion gain}

In the low-gain regime, where the effect of SPM and XPM can be neglected, and assuming a homogeneous periodic poling, the PDC equations of motion (6a) and (6b) are defined solely by $\Delta \beta_{\mathrm{s}}, \Delta \beta_{\mathrm{i}}$ and $\beta_{\mathrm{p}}(z=0, \omega)$, as well as the PDC coupling strength $\gamma_{\mathrm{PDC}}$. Integrating the EOMs allows us to compute the quantity $\kappa$, as defined in Eq. (5), which, as we have explained in Sec. II B, is independent of

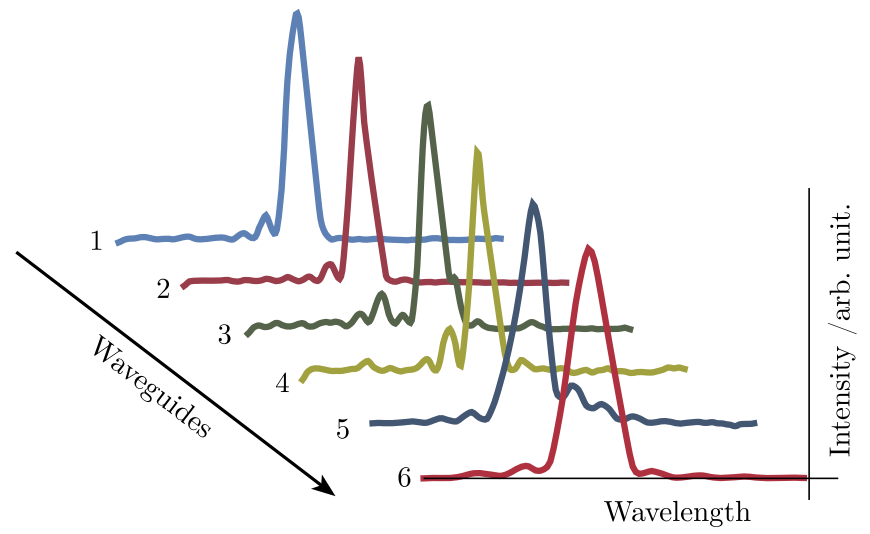

FIG. 14. Phase-matching profiles of different waveguides in the same chip. We observe a variability of the shape of the phasematching profiles, which can be caused by periodic poling inhomogeneity.

the seeding and detection efficiency, using $\gamma_{\mathrm{PDC}}$ as a free fitting parameter. From the results in Appendix B, one can easily show that $\kappa$ is linearly proportional to $\gamma_{\mathrm{PDC}}$ in the low-gain regime. In the high-gain regime, the relation between $\kappa$ and $\gamma_{\mathrm{PDC}}$ becomes nonlinear, but it remains monotonically increasing. This monotonic dependence allows us to use $\kappa$ as a robust proxy to derive the PDC coupling strength $\gamma_{\mathrm{PDC}}$ from experimental intensity ratios.

In our experimental demonstration, we fit the predictions of our model to the experimental values of $\kappa$ as a function of pump pulse energy, as illustrated in Fig. 6. In order to mitigate the effect of measurement noise, we smooth the spectral distributions using a Gaussian kernel with a standard deviation of $0.35 \mathrm{~nm}$ before taking their maxima. The best-fit parameter was $\gamma_{\mathrm{PDC}}=28 \mathrm{~W}^{-1 / 2} / \mathrm{m}^{-1}$.

\section{Cross-phase modulation}

Cross-phase modulation of the narrow-band seed by the broadband pump appears as a spectral broadening of the former. In our seeded measurements, this effect appears as a broadband pedestal surrounding the $\mathrm{cw}$ component of the same-mode TFs. According to our simulations of $U_{\mathrm{b}}^{\mathrm{s}, \mathrm{s} / \mathrm{i}, \mathrm{i}}\left(\omega_{\text {out }}, \omega_{\mathrm{in}}\right)$, the amplitude is constant along a contour where $\omega_{\text {in }}+\omega_{\text {out }}$ is constant, and its magnitude is proportional to the power of the pump. As mentioned in the previous Appendixes (e.g., Appendix B), the same-mode TFs contain another broadband component due to cascaded PDC. Our simulations show that the cascaded PDC amplitude scales linearly with the pump power, and it exhibits a $\pi$ phase jump around the central phase-matching wavelength [this is also readily seen by examining the analytical result in Eq. (B21)]. Because of this nontrivial spectral phase, the XPM and PDC contributions interfere either constructively or destructively at different frequencies, resulting in an emission spectrum shaped like a Fano 


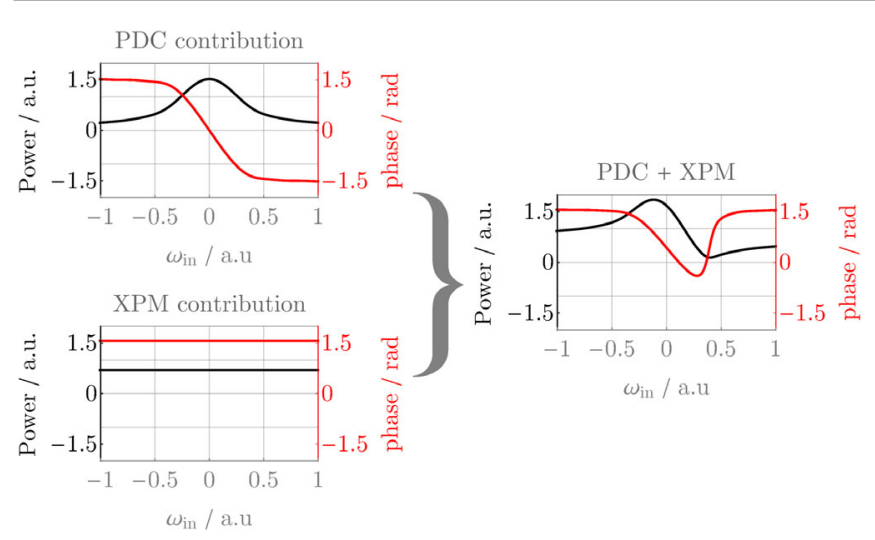

FIG. 15. Asymmetric magnitude of the TF "cut" $U_{(b)}^{s, s}(\omega, \omega)$ due to interference between PDC and XPM. Top-left panel: Simulated cut with only PDC. Bottom-left panel: Simulated cut with only XPM. Right panel: Simulated cut with PDC and XPM.

resonance [38], as illustrated in Fig. 15. The asymmetry of this spectrum allows us to accurately estimate the XPM coupling strength in relation to the PDC coupling strength. In Fig. 4, we use diagonal cuts of the same-mode TFs at the lowest power (in order to avoid other nonlinear phenomena) and find the XPM interaction strength $\gamma_{\mathrm{XPM}, \mathrm{s}(\mathrm{i})}$ that best fits their asymmetric "tails." The best-fit parameters are $\gamma_{\mathrm{XPM}, \mathrm{s}}=0.16 \mathrm{~W}^{-1} \mathrm{~m}^{-1}$ and $\gamma_{\mathrm{XPM}, \mathrm{i}}=0.06 \mathrm{~W}^{-1} \mathrm{~m}^{-1}$.

\section{Self-phase modulation of the pump}

The effect of SPM of the pump is visible in the high-gain regime: The cross-mode TFs show a clear broadening, as well as splitting of the pump function. Also, the same-mode TFs lose their symmetry, which is consistent with a chirped pump, as illustrated in Fig. 5.

Assuming that dispersion of the pump is negligible, its evolution along the waveguide is given by the differential equation (7). Having measured $\beta_{\mathrm{p}}(z=0, \omega)$, the SPM interaction strength $\gamma_{\mathrm{SPM}}$ is the only fitting parameter left, which determines the evolution of the pump spectral amplitude along the waveguide. To obtain the value of $\gamma_{\text {SPM }}$, we take the complete signal or idler EOMs (6a) and (6b), with all the other parameters fitted using the low-gain regime measurements described above, and we find the value of the SPM interaction strength that best fits the highgain TFs according to the error metric defined in Eq. (11). The best-fit parameter is $\gamma_{\mathrm{SPM}}=0.56 \mathrm{~W}^{-1} \mathrm{~m}^{-1}$.

\section{APPENDIX E: ASYMMETRY IN THE CROSS-PHASE MODULATION COEFFICIENTS}

In this Appendix, we provide an analysis of the way in which waves propagating in a material can experience different nonlinear effects because of their polarization relative to a strong pump. Suppose we have a pump wave with central frequency $\bar{\omega}_{\mathrm{p}}$ and a signal wave with central frequency $\bar{\omega}_{\text {s }}$ polarized in the same direction, say, the $y$ direction. Then, the polarization term responsible for the XPM will be [47]

$$
P_{y}\left(\bar{\omega}_{\mathrm{s}}\right)=\epsilon_{0} \chi_{y y y y}^{(3)} E_{y}\left(\bar{\omega}_{\mathrm{s}}\right) E_{y}\left(\bar{\omega}_{\mathrm{p}}\right) E_{y}\left(-\bar{\omega}_{\mathrm{p}}\right)+\ldots,
$$

where ... are terms obtained by permuting the different fields appearing in the first term. The fields associated with the three frequencies $\left(\bar{\omega}_{\mathrm{s}}, \bar{\omega}_{\mathrm{p}},-\bar{\omega}_{\mathrm{p}}\right)$ can be combined in any order, giving $3 !=6$ different terms. Thus, in all, we would have

$$
P_{y}\left(\bar{\omega}_{\mathrm{s}}\right)=6 \epsilon_{0} \chi_{\text {yyyy }}^{(3)} E_{y}\left(\bar{\omega}_{\mathrm{s}}\right) E_{y}\left(\bar{\omega}_{\mathrm{p}}\right) E_{y}\left(-\bar{\omega}_{\mathrm{p}}\right) .
$$

Now, suppose the pump is in the $y$ direction, but the idler is in the $x$ direction. Then, one of the terms contributing to the cross-phase modulation would be

$$
P_{x}\left(\bar{\omega}_{\mathrm{i}}\right)=\epsilon_{0} \chi_{x x y y}^{(3)} E_{x}\left(\bar{\omega}_{\mathrm{i}}\right) E_{y}\left(\bar{\omega}_{\mathrm{p}}\right) E_{y}\left(-\bar{\omega}_{\mathrm{p}}\right)+\ldots
$$

For this particular $\chi^{(3)}$ component, we could put the fields at $\bar{\omega}_{\mathrm{p}}$ and $-\bar{\omega}_{\mathrm{p}}$ in other orders (2! permutations), so from this particular component, we would expect

$$
P_{x}\left(\bar{\omega}_{\mathrm{i}}\right)=2 \epsilon_{0} \chi_{x x y y}^{(3)} E_{x}\left(\bar{\omega}_{\mathrm{i}}\right) E_{y}\left(\bar{\omega}_{\mathrm{p}}\right) E_{y}\left(-\bar{\omega}_{\mathrm{p}}\right) .
$$

However, there will be other tensor components involving $x$ 's and $y$ 's. In particular, we can expect a $\chi_{x y y x}^{(3)}$ and a $\chi_{x y x y}^{(3)}$. In each one of these components, there will be two permutations of the fields at $\bar{\omega}_{\mathrm{p}}$ and $-\bar{\omega}_{\mathrm{p}}$ that share the same Cartesian component, so in all

$$
\begin{aligned}
P_{x}\left(\bar{\omega}_{\mathrm{i}}\right)= & 2 \epsilon_{0} \chi_{x x y y}^{(3)} E_{x}\left(\bar{\omega}_{\mathrm{i}}\right) E_{y}\left(\bar{\omega}_{\mathrm{p}}\right) E_{y}\left(-\bar{\omega}_{\mathrm{p}}\right) \\
& +2 \epsilon_{0} \chi_{x y y x}^{(3)} E_{y}\left(\bar{\omega}_{\mathrm{p}}\right) E_{y}\left(-\bar{\omega}_{\mathrm{p}}\right) E_{x}\left(\bar{\omega}_{\mathrm{i}}\right) \\
& +2 \epsilon_{0} \chi_{x y x y}^{(3)} E_{y}\left(\bar{\omega}_{\mathrm{p}}\right) E_{x}\left(\bar{\omega}_{\mathrm{i}}\right) E_{y}\left(-\bar{\omega}_{\mathrm{p}}\right) \\
= & 2 \epsilon_{0}\left(\chi_{x x y y}^{(3)}+\chi_{x y y x}^{(3)}+\chi_{x y x y}^{(3)}\right) \\
& \times E_{x}\left(\bar{\omega}_{\mathrm{i}}\right) E_{y}\left(\bar{\omega}_{\mathrm{p}}\right) E_{y}\left(-\bar{\omega}_{\mathrm{p}}\right) .
\end{aligned}
$$

which are for different polarizations. For the same polarization, we have Eq. (E2). So,

$$
P_{y}\left(\bar{\omega}_{\mathrm{s}}\right)=6 \epsilon_{0} \chi_{\text {yyyy }}^{(3)} E_{y}\left(\bar{\omega}_{\mathrm{s}}\right) E_{y}\left(\bar{\omega}_{\mathrm{p}}\right) E_{y}\left(-\bar{\omega}_{\mathrm{p}}\right) \quad(\text { same pol })
$$

$$
\begin{aligned}
P_{x}\left(\bar{\omega}_{\mathrm{s}}\right)= & 2 \epsilon_{0}\left(\chi_{x x y y}^{(3)}+\chi_{x y y x}^{(3)}+\chi_{x y x y}^{(3)}\right) \\
& \times E_{x}\left(\bar{\omega}_{\mathrm{s}}\right) E_{y}\left(\bar{\omega}_{\mathrm{p}}\right) E_{y}\left(-\bar{\omega}_{\mathrm{p}}\right) \quad(\text { diff pol }) .
\end{aligned}
$$

If one assumes the following symmetry in the third-order susceptibility (satisfied, for instance, by an isotropic medium), 


$$
\chi_{y y y y}^{(3)}=\chi_{x x y y}^{(3)}+\chi_{x y y x}^{(3)}+\chi_{x y x y}^{(3)}
$$

so

$$
\begin{aligned}
P_{y}\left(\bar{\omega}_{\mathrm{s}}\right)= & 6 \epsilon_{0}\left(\chi_{x x y y}^{(3)}+\chi_{x y y x}^{(3)}+\chi_{x y x y}^{(3)}\right) \\
& \times E_{y}\left(\bar{\omega}_{\mathrm{s}}\right) E_{y}\left(\bar{\omega}_{\mathrm{p}}\right) E_{y}\left(-\bar{\omega}_{\mathrm{p}}\right) \quad(\text { same pol }), \\
P_{x}\left(\bar{\omega}_{\mathrm{i}}\right)= & 2 \epsilon_{0}\left(\chi_{x x y y}^{(3)}+\chi_{x y y x}^{(3)}+\chi_{x y x y}^{(3)}\right) \\
& \times E_{x}\left(\bar{\omega}_{\mathrm{i}}\right) E_{y}\left(\bar{\omega}_{\mathrm{p}}\right) E_{y}\left(-\bar{\omega}_{\mathrm{p}}\right) \quad(\text { diff pol }),
\end{aligned}
$$

differing precisely by a factor of 3 . To the best of our knowledge, the tensor components of the third-order nonlinear susceptibility of PPKTP have not been reported in the literature; however, this simple calculation provides a plausible argument for the approximate factor of 3 found between the different cross-phase modulation constants of the signal and idler fields.

[1] P. J. Mosley, J. S. Lundeen, B. J. Smith, P. Wasylczyk, A. B. U'Ren, C. Silberhorn, and I. A. Walmsley, Heralded Generation of Ultrafast Single Photons in Pure Quantum States, Phys. Rev. Lett. 100, 133601 (2008).

[2] A. Eckstein, A. Christ, P. J. Mosley, and C. Silberhorn, Highly Efficient Single-Pass Source of Pulsed Single-Mode Twin Beams of Light, Phys. Rev. Lett. 106, 013603 (2011).

[3] M. M. Weston, H. M. Chrzanowski, S. Wollmann, A. Boston, J. Ho, L. K. Shalm, V. B. Verma, M. S. Allman, S. W. Nam, R. B. Patel et al., Efficient and Pure Femtosecond-Pulse-Length Source of Polarization-Entangled Photons, Opt. Express 24, 10869 (2016).

[4] G. Harder, T. J. Bartley, A. E. Lita, S. W. Nam, T. Gerrits, and C. Silberhorn, Single-Mode Parametric-Down-Conversion States with 50 Photons as a Source for Mesoscopic Quantum Optics, Phys. Rev. Lett. 116, 143601 (2016).

[5] D. Fukuda, G. Fujii, T. Numata, K. Amemiya, A. Yoshizawa, H. Tsuchida, H. Fujino, H. Ishii, T. Itatani, S. Inoue et al., Titanium-Based Transition-Edge Photon Number Resolving Detector with 98\% Detection Efficiency with Index-Matched Small-Gap Fiber Coupling, Opt. Express 19, 870 (2011).

[6] A. Zavatta, S. Viciani, and M. Bellini, Quantum-toClassical Transition with Single-Photon-Added Coherent States of Light, Science 306, 660 (2004).

[7] J. Wenger, R. Tualle-Brouri, and P. Grangier, Non-Gaussian Statistics from Individual Pulses of Squeezed Light, Phys. Rev. Lett. 92, 153601 (2004).

[8] A. P. Lund, A. Laing, S. Rahimi-Keshari, T. Rudolph, J. L. O'Brien, and T. C. Ralph, Boson Sampling from a Gaussian State, Phys. Rev. Lett. 113, 100502 (2014).

[9] C. S. Hamilton, R. Kruse, L. Sansoni, S. Barkhofen, C. Silberhorn, and I. Jex, Gaussian Boson Sampling, Phys. Rev. Lett. 119, 170501 (2017).

[10] A. P. Lund, S. Rahimi-Keshari, and T. C. Ralph, Exact Boson Sampling Using Gaussian Continuous-Variable Measurements, Phys. Rev. A 96, 022301 (2017).
[11] J. Huh, G. G. Guerreschi, B. Peropadre, J. R. McClean, and A. Aspuru-Guzik, Boson Sampling for Molecular Vibronic Spectra, Nat. Photonics 9, 615 (2015).

[12] C. K. Law, I. A. Walmsley, and J. H. Eberly, Continuous Frequency Entanglement: Effective Finite Hilbert Space and Entropy Control, Phys. Rev. Lett. 84, 5304 (2000).

[13] W. Wasilewski, A. I. Lvovsky, K. Banaszek, and C. Radzewicz, Pulsed Squeezed Light: Simultaneous Squeezing of Multiple Modes, Phys. Rev. A 73, 063819 (2006).

[14] A. I. Lvovsky, W. Wasilewski, and K. Banaszek, Decomposing a Pulsed Optical Parametric Amplifier into Independent Squeezers, J. Mod. Opt. 54, 721 (2007).

[15] A. Christ, B. Brecht, W. Mauerer, and C. Silberhorn, Theory of Quantum Frequency Conversion and Type-II Parametric Down-Conversion in the High-Gain Regime, New J. Phys. 15, 053038 (2013).

[16] M. L. Sundheimer, C. Bosshard, E. W. Van Stryland, G. I. Stegeman, and J. D. Bierlein, Large Nonlinear Phase Modulation in Quasi-Phase-Matched KTP Waveguides as a Result of Cascaded Second-Order Processes, Opt. Lett. 18, 1397 (1993).

[17] P. Cutipa, K. Yu. Spasibko, and M. V. Chekhova, Direct Measurement of the Coupled Spatiotemporal Coherence of Parametric Down-Conversion under Negative GroupVelocity Dispersion, Opt. Lett. 45, 3581 (2020).

[18] C. Liberale, I. Cristiani, V. Degiorgio, M. Marangoni, G. Galzerano, and R. Ramponi, Cross-Phase Modulation Due to a Cascade of Quadratic Interactions in a PPLN Waveguide, IEEE J. Sel. Top. Quantum Electron. 12, 405 (2006).

[19] A. Christ, K. Laiho, A. Eckstein, K. N. Cassemiro, and C. Silberhorn, Probing Multimode Squeezing with Correlation Functions, New J. Phys. 13, 033027 (2011).

[20] N. Quesada and J. E. Sipe, Effects of Time Ordering in Quantum Nonlinear Optics, Phys. Rev. A 90, 063840 (2014).

[21] N. Quesada, G. Triginer, M. D. Vidrighin, and J. E. Sipe, Theory of High-Gain Twin-Beam Generation in Waveguides: From Maxwell's Equations to Efficient Simulation, Phys. Rev. A 102, 033519 (2020).

[22] J. L. O'Brien, Optical Quantum Computing, Science 318, 1567 (2007).

[23] B. Brecht, A. Eckstein, A. Christ, H. Suche, and C. Silberhorn, From Quantum Pulse Gate to Quantum Pulse Shaper-Engineered Frequency Conversion in Nonlinear Optical Waveguides, New J. Phys. 13, 065029 (2011).

[24] M. Liscidini and J. E. Sipe, Stimulated Emission Tomography, Phys. Rev. Lett. 111, 193602 (2013).

[25] A. Eckstein, G. Boucher, A. Lemaître, P. Filloux, I. Favero, G. Leo, J. E. Sipe, M. Liscidini, and S. Ducci, High-Resolution Spectral Characterization of Two Photon States via Classical Measurements, Laser Photonics Rev. 8, L76 (2014).

[26] B. Fang, O. Cohen, M. Liscidini, J. E. Sipe, and V. O. Lorenz, Fast and Highly Resolved Capture of the Joint Spectral Density of Photon Pairs, Optica 1, 281 (2014).

[27] B. Fang, M. Liscidini, J. E. Sipe, and V. O. Lorenz, Multidimensional Characterization of an Entangled Photon-Pair Source via Stimulated Emission Tomography, Opt. Express 24, 10013 (2016). 
[28] L. A. Rozema, C. Wang, D. H. Mahler, A. Hayat, A. M. Steinberg, J. E. Sipe, and M. Liscidini, Characterizing an Entangled-Photon Source with Classical Detectors and Measurements, Optica 2, 430 (2015).

[29] I. Jizan, B. Bell, L. G. Helt, A. C. Bedoya, C. Xiong, and B. J. Eggleton, Phase-Sensitive Tomography of the Joint Spectral Amplitude of Photon Pair Sources, Opt. Lett. 41, 4803 (2016).

[30] V. Ansari, G. Harder, M. Allgaier, B. Brecht, and C. Silberhorn, Temporal-Mode Measurement Tomography of a Quantum Pulse Gate, Phys. Rev. A 96, 063817 (2017).

[31] E. A. Goldschmidt, F. Piacentini, I. R. Berchera, S. V. Polyakov, S. Peters, S. Kück, G. Brida, I. P. Degiovanni, A. Migdall, and M. Genovese, Mode Reconstruction of a Light Field by Multiphoton Statistics, Phys. Rev. A 88, 013822 (2013).

[32] I. A. Burenkov, A. K. Sharma, T. Gerrits, G. Harder, T. J. Bartley, C. Silberhorn, E. A. Goldschmidt, and S. V. Polyakov, Full Statistical Mode Reconstruction of a Light Field via a Photon-Number-Resolved Measurement, Phys. Rev. A 95, 053806 (2017).

[33] D. N. Klyshko, Use of Two-Photon Light for Absolute Calibration of Photoelectric Detectors, Sov. J. Quantum Electron. 10, 1112 (1980).

[34] T. Lunghi, B. Korzh, B. Sanguinetti, and H. Zbinden, Absolute Calibration of Fiber-Coupled Single-Photon Detector, Opt. Express 22, 18078 (2014).

[35] W. P. Grice and I. A. Walmsley, Spectral Information and Distinguishability in Type-II Down-Conversion with a Broadband Pump, Phys. Rev. A 56, 1627 (1997).

[36] N. Quesada and J. E. Sipe, Time-Ordering Effects in the Generation of Entangled Photons Using Nonlinear Optical Processes, Phys. Rev. Lett. 114, 093903 (2015).
[37] M.-D. Vidrighin, Quantum Optical Measurements for Practical Estimation and Information Thermodynamics, Ph.D Thesis, Imperial College London, 2017.

[38] U. Fano, Effects of Configuration Interaction on Intensities and Phase Shifts, Phys. Rev. 124, 1866 (1961).

[39] J. H. Eberly, Schmidt Analysis of Pure-State Entanglement, Laser Phys. 16, 921 (2006).

[40] T. S. Iskhakov, K. Yu. Spasibko, M. V. Chekhova, and G. Leuchs, Macroscopic Hong-Ou-Mandel Interference, New J. Phys. 15, 093036 (2013).

[41] I. V. Dyakonov, P. R. Sharapova, T. S. Iskhakov, and G. Leuchs, Direct Schmidt Number Measurement of HighGain Parametric Down Conversion, Laser Phys. Lett. 12, 065202 (2015).

[42] M. Cooper, L. J. Wright, C. Söller, and B. J. Smith, Experimental Generation of Multi-photon Fock States, Opt. Express 21, 5309 (2013).

[43] P. R. Sharapova, G. Frascella, M. Riabinin, A. M. Pérez, O. V. Tikhonova, S. Lemieux, R. W. Boyd, G. Leuchs, and M. V. Chekhova, Properties of Bright Squeezed Vacuum at Increasing Brightness, Phys. Rev. Research 2, 013371 (2020).

[44] P. J. Mosley, Generation of Heralded Single Photons in Pure Quantum States, Ph.D thesis, University of Oxford, 2007.

[45] M. M. Fejer, G. A. Magel, D. H. Jundt, and R. L. Byer, Quasi-Phase-Matched Second Harmonic Generation: Tuning and Tolerances, IEEE J. Quantum Electron. 28, 2631 (1992).

[46] S. Guo, Y. Ge, Y. Han, J. He, and J. Wang, Investigation of Optical Inhomogeneity of MgO: PPLN Crystals for Frequency Doubling of $1560 \mathrm{~nm}$ Laser, Opt. Commun. 326, 114 (2014).

[47] R. W. Boyd, Nonlinear Optics (Academic Press, New York, 2019). 